\title{
A reflection-based efficient wavefield inversion
}

\author{
Chao Song, Tariq Alkhalifah \\ King Abdullah University of Science and Technology (KAUST), \\ Physical Sciences and Engineering Division, \\ Thuwal, Saudi Arabia, 23955
}

(March 25, 2021)

Running head: Reflection-based EWI

\begin{abstract}
Full-waveform inversion (FWI) is popularly used to obtain a high-resolution subsurface velocity model. However, it requires either a good initial velocity model or low-frequency data to mitigate the cycle-skipping issue. Reflection-waveform inversion (RWI) uses a migration/demigration process to retrieve a background model that can be used as a good initial velocity in FWI. The drawback of the conventional RWI is that it requires the use of a least-squares migration, which is often computationally expensive, and is still prone to cycle skipping at far offsets. To improve the computational efficiency and overcome the cycle skipping in the original RWI, we incorporate it into a recently introduced method called efficient wavefield inversion (EWI) by inverting for the Born scattered wavefield instead of the wavefield itself. In this case, we use perturbation-related secondary sources in the modified source function. Unlike conventional RWI, the perturbations are calculated naturally as part of the calculation of the scattered wavefield in an efficient way. As the sources in the reflection-based EWI (REWI) are located in the subsurface, we are able to update the background model along the reflection wave path. In the background velocity
\end{abstract}


inversion, we calculate the background perturbation by a deconvolution process at each frequency. After obtaining the REWI inverted velocity model, a sequential FWI or EWI is needed to obtain a high resolution model. We demonstrate the validity of the proposed approach using synthetic data generated from a section of the Sigsbee2A model. To further demonstrate the effectiveness of the proposed approach, we test it on an ocean bottom cable (OBC) dataset from the North Sea. We find that the proposed methodology leads to improved velocity models as evidenced by flatter angle gathers. 


\section{INTRODUCTION}

Full-waveform inversion (FWI) has shown great potential in retrieving high-resolution velocity models of the earth (Tarantola, 1984). However, FWI has specific requirements on the initial velocity and recorded data for convergence (Mora, 1989). It, specifically, suffers from the cycle-skipping problem if the data lack low-frequency components or the available starting model for FWI is poor. Researchers try to resolve this issue from two perspectives. One perspective is to build a good initial velocity model for FWI. For example, tomography uses the traveltime information in the data to recover a smooth velocity model (Clement et al., 2001; Biondi and Almomin, 2014). Alternatively, migration velocity analysis (MVA) aims at recovering the kinematic features of the model for imaging (Biondi and Symes, 2004; Symes, 2008). We can also transform the recorded data into other domains or extract specific features to retrieve the low-wavenumber updates, like waveform inversion in the Laplace domain (Shin and Cha, 2008), seismic envelope inversion (Chi et al., 2014; Wu et al., 2014; Liu et al., 2020), phase-based waveform inversion (Bozdă̆ et al., 2011; Choi and Alkhalifah, 2013). In addition, some researchers have used extrapolated low-frequency data to recover the low-wavenumber model components (Li and Demanet, 2016; Ovcharenko et al., 2019). Recently, reflection-waveform inversion (RWI) has become a popular method for background model retrieval, as it is capable of updating a smooth background model along reflection wave paths (Xu et al., 2012; Zhou et al., 2012). In RWI, Born modeling is used to separate the tomography and migration components in the velocity gradient (Wang et al., 2018; Li and Alkhalifah, 2020; Yao et al., 2020), and the tomography components are used to correct errors in the background velocity (Yao et al., 2019). Traveltime-based reflection RWI was proposed and demonstrated its effectiveness on marine seismic data (Wang et al., 2017; Sun et al., 2017). However, it is often expensive mainly because we often need 
to implement a least-squares reverse time migration (LSRTM) to obtain a true-amplitude image at each iteration. To improve the computational efficiency, a new objective function was proposed to separate the data into the scattering and background components (Alkhalifah and $\mathrm{Wu}, 2016)$. A simultaneous inversion for the background and perturbation models using this new objective function was proposed by Wu and Alkhalifah (2015).

The other perspective to mitigate the cycle-skipping issue is to formulate new objective functions for waveform inversion. Conventional FWI minimizes the data misfit between the recorded and predicted data pixel by pixel. Wavefield reconstruction inversion (WRI) relaxes the wave equation accuracy by using it as a regularization term. By doing so, the data fitting is enhanced to avoid cycle skipping (Van Leeuwen and Herrmann, 2013; van Leeuwen and Herrmann, 2015). Due to the size of wavefields, WRI is naturally performed in the frequency domain, and we often need to solve an augmented wave equation many times per frequency to build a credible model. To reduce the computational cost, an efficient wavefield inversion (EWI) method was proposed (Alkhalifah and Song, 2019). In EWI, the modeling operator is kept stationary for each frequency by using a modified source function as an independent parameter. The modified source function captures the secondary sources (perturbations) allowing the inversion of a more complete wavefield.

WRI and EWI can reduce the cycle-skipping problem, but they are also able to provide both low- and high-wavenumber updates (van Leeuwen and Herrmann, 2015; Song and Alkhalifah, 2020). However, they are not completely immune to cycle skipping when the initial velocity is far away from the true one, regardless of how we control the weighting parameter in the objective function (Alkhalifah and Song, 2019). Thus, we develop a new method to estimate a good background model to resolve the partial cycle-skipping issue in WRI and EWI. We extend the original EWI by separating the reconstructed wavefield into 
a background wavefield and a scattered wavefield, utilizing the approach of Alkhalifah and $\mathrm{Wu}(2016)$ that naturally combines FWI and RWI. In this case, two regularization terms corresponding to the background wave equation and the Born scattered wave equation are used. Specifically, we formulate sub-problems to calculate the perturbation and background models sequentially. We calculate the perturbation model prior to the background model without the need to perform LSRTM.

The main contribution of this paper is to develop a reflection-based efficient wavefield inversion (REWI) to improve the efficiency and mitigate the cycle-skipping issue found in the original RWI. This method does not require an expensive LSRTM process within each iteration. The perturbation model acts as secondary sources generating the scattered wavefield and can be easily estimated by reconstructing the scattered wavefield. Even though the estimated perturbation may deviate from its true depth in the early stages of the background model inversion, REWI is still able to match the reflection data by relaxing the requirement for the scattered wavefield to satisfy the Born scattered wave equation. Similar to the original EWI, we are able to calculate the background model update directly. After obtaining a reasonable background model, a sequential FWI or EWI (applied after REWI) will inject detailed information to build a high-resolution model. We test the proposed approach on synthetic data that lacks frequencies below $5 \mathrm{~Hz}$ generated from a section of the Sigsbee2A model. A test on the North Sea Volve OBC dataset demonstrates the effectiveness of the proposed method. 


\section{THEORY}

\section{Efficient wavefield inversion}

We simulate wave propagation using a frequency-domain acoustic wave equation, with a constant density, given by:

$$
\frac{\omega^{2}}{v^{2}} u(\mathbf{x}, \omega)+\nabla^{2} u(\mathbf{x}, \omega)=f\left(\mathbf{x}_{\mathbf{s}}, \omega\right)
$$

where $\omega$ represents the angular frequency, $u(\mathbf{x}, \omega)$ denotes the pressure wavefield, and $\mathbf{x}_{\mathbf{s}}$ represents the coordinates of the source. The source function $f\left(\mathbf{x}_{\mathbf{s}}, \omega\right)$ is given in the frequency domain, and $v$ denotes the velocity. Equation 1 can be expressed in a compact form as:

$$
L(m) u(\mathbf{x}, \omega)=f\left(\mathbf{x}_{\mathbf{s}}, \omega\right)
$$

where $L(m)=\omega^{2} m+\nabla^{2}$ is the impedance matrix, which is also referred to as the modeling operator. Here, we use $m$ to represent the squared slowness field, and this is the target parameter in wavefield-reconstruction based methods, like WRI and EWI. In classic frequency domain modeling, equation 2 can be solved efficiently with LU decomposition. In conventional frequency-domain FWI, we use the wavefield from such modeling to measure the misfit between the recorded and predicted data under the constraint of the wave equation:

$$
E(m)_{F W I}=\frac{1}{2} \sum_{i}\left\|d_{i}-C u_{i}\right\|_{2}^{2}, \text { such that } L(m) u_{i}=f_{i},
$$

where $i$ is the source index, and $d_{i}$ denotes the recorded data. $C$ is a mapping operator extracting the wavefield at the sensor locations. The conventional FWI is a highly non-linear optimization problem, as the wavefield $u$ depends nonlinearly on the model parameters. The velocity gradient of conventional FWI is calculated by cross correlation of the background 
wavefield with the back-propagated residual wavefield. This process is often computationally expensive and requires a large amount of memory to calculate and store the background wavefields. EWI was proposed to solve the problems of conventional FWI, and its objective function is given by:

$$
E\left(u_{i}, f_{e i}\right)=\frac{1}{2} \sum_{i}\left\|d_{i}-C u_{i}\right\|_{2}^{2}+\frac{\alpha^{2}}{2}\left\|L_{0} u_{i}-f_{e i}\right\|_{2}^{2},
$$

where $L_{0}$ is the modeling operator with the background model, $f_{e}$ is the modified source function, and $\alpha^{2}$ is a weighting factor used to balance the data fitting goal with the need to satisfy the background wave equation. A relatively small $\alpha^{2}$ will enhance the data-fitting term to reduce the cycle-skipping issue. However, when the initial velocity is far from the true one and the low frequency components in the data are missing, EWI is not able to completely overcome the cycle-skipping issue (Alkhalifah and Song, 2019). That is because when the initial velocity is far from the true velocity, the wavefield can not be accurately reconstructed.

Reflection waveform inversion (RWI) provides smooth updates of the background velocity if we have the ture-amplitude reflectivity model (Yao and Wu, 2017). Alkhalifah and Wu (2016) use a new objective function to invert for the perturbation and background velocity models. This new objective function separates the total wavefield $u$ into the background wavefield $u_{0}$ and the scattered wavefield $\delta u$, given by:

$$
E\left(\delta m_{0}, \delta m\right)_{R W I}=\frac{1}{2} \sum_{i}\left\|C u_{0 i}+C \delta u_{i}-d_{i}\right\|_{2}^{2} \text { such that } L_{0} u_{0 i}=f_{i} \text { and } L_{0} \delta u_{i}=s_{i}
$$

where $s_{i}=-\omega^{2} \delta m u_{0}$ represents secondary sources in the subsurface under the Born approximation, $\delta m$ is the reflectivity-like perturbation model, and $\delta m_{0}$ is the background model perturbation between the desired good background model and the available poor initial background model. With the help of this new objective function, we can match the 
direct arrivals generated from the true sources and reflections generated from the perturbations simultaneously. To incorporate the work of Alkhalifah and Wu (2016) into the EWI framework, we extend the reconstructed wavefield into two parts: the background wavefield $u_{0}$ and the scattered wavefield $\delta u$. In this case, we include two regularization terms corresponding to $u_{0}$ and $\delta u$ satisfying the wave equation. Therefore, the extended EWI objective function can be stated as:

$$
E\left(u_{0 i}, \delta u_{i}, s_{e i}\right)=\frac{1}{2} \sum_{i}\left\|C u_{0 i}+C \delta u_{i}-d_{i}\right\|_{2}^{2}+\frac{\alpha^{2}}{2}\left\|L_{0} u_{0 i}-f_{i}\right\|_{2}^{2}+\frac{\epsilon^{2}}{2}\left\|L_{0} \delta u_{i}-s_{e i}\right\|_{2}^{2},
$$

where $s_{e i}$ is the modified secondary source function and is equal to $s_{i}$ at the first outer iteration. As we will see later, this source function will provide smooth background velocity updates along reflection wave paths, similar to RWI. The weighting factor $\epsilon^{2}$ controls how much the scattered wavefield needs to satisfy the Born scattered wave equation. Like $\alpha^{2}$ in equation 4 , a proper $\epsilon^{2}$ should balance the magnitude of the third term with the other two terms in equation 6 (Song and Alkhalifah, 2020). If we focus on minimizing the reflection data misfit like RWI, the reflection-based EWI (REWI) objective function can be stated as:

$$
E\left(\delta u_{i}, s_{e i}\right)=\frac{1}{2} \sum_{i}\left\|C u_{0 i}+C \delta u_{i}-d_{i}\right\|_{2}^{2}+\frac{\epsilon^{2}}{2}\left\|L_{0} \delta u_{i}-s_{e i}\right\|_{2}^{2}, \text { such that } L_{0} u_{0 i}=f_{i}
$$

Analogously to the original EWI idea, this new objective function aims to minimize the misfit in the scattered wavefield, which partially satisfies the Born scattered wave equation. The scattered wavefield $\delta u$ can be solved using a modified augmented wave equation given by:

$$
\left(\begin{array}{c}
\epsilon L_{0} \\
C
\end{array}\right) \delta u_{i}=\left(\begin{array}{c}
\epsilon s_{e i} \\
d_{i}-C u_{i 0}
\end{array}\right)
$$

As $s_{e i}$ starts with $s_{i}$ at each frequency, we will need to calculate the perturbation $\delta m$ prior to updating the background velocity model. Next, we discuss the inversion of $\delta m$. 


\section{Inversion of the perturbation model}

To have a reconstruction of the scattered wavefield $\delta u$ using equation 8 , we need to subtract the data corresponding to the background wavefield $u_{0}$ from the recorded data. This step is equivalent to muting the direct waves for the time-domain data. Theoretically, this background wavefield should correspond to a good background velocity model, so we cannot calculate it directly using the background wave equation, which may correspond to a poor initial velocity. Luckily, we can still use the augmented wave equation to reconstruct the background wavefield, even with a poor initial velocity model. After we get the background wavefield $u_{0}$ at each frequency, we set $\delta m=0$ at the first iteration, which means $s=0$. Even under this condition of $s=0$, we can still use equation 8 to initially reconstruct the scattered wavefield $\delta u$. This is equivalent to microseismic source estimation, because $s_{i}$ corresponds to secondary sources in the subsurface (Song and Alkhalifah, 2019). Following the Born approximation, the wave equation connects $\delta m, \delta u$, and $u_{0}$ as follows (Zhang and Duan, 2012):

$$
L_{0} \delta u=-\omega^{2} \delta m u_{0}
$$

The squared slowness perturbation $\delta m$ can be evaluated using:

$$
\delta m=-\Re\left(\sum_{i} \frac{L_{0} \delta u_{i}}{\omega^{2} u_{0 i}}\right) \approx-\Re\left(\sum_{i} \frac{\left(L_{0} \delta u_{i}\right) u_{0 i}^{*}}{\omega^{2} u_{0 i} u_{0 i}^{*}+\lambda}\right) .
$$

To further improve the accuracy of the scattered wavefield $\delta u$, we insert the calculated $\delta m$ back into equation 8 , and repeat equations 9 and 10 . In the perturbation model inversion, we do not update the modified source function $s_{e i}$, so it is equivalent to $s_{i}$. As we implement our method in the frequency domain, we calculate the perturbation model $\delta m_{\text {ifre }}$ at each frequency, and stack them over the full frequency band to get the final perturbation model $\delta m$ to reduce the artifacts associated with the monofrequency inversion. The 

frequency band $\left[f_{\min }: d f: f_{\max }\right]$; Weighting factors $\alpha^{2}$ and $\epsilon^{2}$.

Output: Inverted perturbation $\delta m$.

$$
\delta m=0 .
$$

for ifre $=\left[f_{\min }: d f: f_{\max }\right]$ do

Reconstruct the background wavefield $u_{0}$ using $\left(\begin{array}{c}\alpha L_{0} \\ C\end{array}\right) u_{0 i}=\left(\begin{array}{c}\alpha f_{i} \\ d_{i}\end{array}\right)$.

Initialize the perturbation at each frequency by setting: $\delta m_{i f r e}=0$.

for $i=1: 2$ do

Reconstruct the scattered wavefield $\delta u$ using $\left(\begin{array}{c}\epsilon L_{0} \\ C\end{array}\right) \delta u=\left(\begin{array}{c}-\epsilon \omega^{2} \delta m_{i f r e} u_{0 i} \\ d_{i}-C u_{0 i}\end{array}\right)$.

Calculate $\delta m_{i f r e}$ at each frequency using $\delta m_{i f r e} \approx-\Re\left(\sum_{i} \frac{\left(L_{0} \delta u_{i}\right) u_{0 i}^{*}}{\omega^{2} u_{0 i} u_{0 i}^{*}+\lambda}\right)$.

\section{end for}

Stack $\delta m_{i f r e}$ over all the frequencies: $\delta m^{(i f r e+1)}=\delta m^{(i f r e)}+\delta m_{i f r e}$.

end for

detailed implementation is outlined in Algorithm 1. During the process of the perturbation model inversion, we do not update the background velocity model. The perturbation model inversion is an essential step in each outer iteration of inverting for the background model. As the background model matures, the quality of $\delta m$ improves as well. The proposed method achieves the perturbation model inversion efficiently by updating it only once per frequency. By comparison, conventional RWI optimizes the true-amplitude image in a least-squares sense until convergence is achieved. Thus, conventional RWI requires a lot more iterations to achieve convergence than the proposed method. We share the algorithm for inverting for the background model in the next section. 
Algorithm 2 The background model inversion in REWI

Input: Recorded data $d$; Initial squared slowness model $m_{0}$, and the true source function $f_{i}$; Selected frequency band; Outer iteration number $N$; Weighting factors $\alpha^{2}$ and $\epsilon^{2}$.

Output: Inverted background model $m_{0}$.

for $k=1: N$; do

Calculate the perturbation $\delta m$ over the full frequency band.

for ifre $=\left[f_{\min }: d f: f_{\max }\right] ;$ do

Initialize the scattered wavefield in each frequency by setting: $\delta u_{i}=0$.

for $i=1: 2 ;$ do

Reconstruct $u_{0 i}$ and update the secondary source function $s_{i}$.

Solve the Born wave equation $L_{0} \delta u_{i}=s_{i}$.

end for

Initialize the modified source function by: $s_{e i}=s_{i}$.

for $i=1: 2 ;$ do

Reconstruct the scattered wavefield $\delta u_{i}$ using $\left(\begin{array}{c}\epsilon L_{0} \\ C\end{array}\right) \delta u_{i}=\left(\begin{array}{c}\epsilon s_{e i} \\ d_{i}-C u_{0 i}\end{array}\right)$.

Update the modified secondary source function $s_{e i}=L_{0} \delta u_{i}$.

end for

Calculate the background perturbation $\delta m_{0}$ and update the operator $L_{0}$.

end for

end for

\section{Inversion of the background model}

After obtaining a reasonable estimation of the perturbation model $\delta m$, we begin our background model inversion step. This new formulation of REWI has two important modifications to the original EWI. The first one is that we are fitting the scattered components of the wavefield to the data instead of the whole wavefield. By doing so, we use mainly the reflection components in the data, like a conventional RWI implementation, to invert for the reflection wavefield's geometrical component. Thus, the resulting velocity update extracted 
from the scattered wavefield wavepaths is smooth, and the background velocity error, like in RWI, will be corrected regardless of the unavailability of the diving waves or low-frequency data. Meanwhile, like FWI, EWI does not utilize reflection wavepath updates. The second one is that we use the modified secondary source $s_{e i}$ instead of $f_{e i}$ to mitigate the cycleskipping issue in the conventional RWI. In order to accurately reconstruct the scattered wavefield, it is important to mitigate the influence of the background wavefield $u_{0}$. Thus, we formulate a sub-problem to solve $u_{0}$ according to equation 6 , given by:

$$
E\left(u_{0 i}\right)=\frac{1}{2} \sum_{i}\left\|C u_{0 i}+C \delta u_{i}-d_{i}\right\|_{2}^{2}+\frac{\alpha^{2}}{2}\left\|L_{0} u_{0 i}-f_{i}\right\|_{2}^{2} \text { such that } L_{0} \delta u_{i}=s_{i} .
$$

As a result, the background wavefield $u_{0}$ satisfies the following linear equation:

$$
\left(\begin{array}{c}
\alpha L_{0} \\
C
\end{array}\right) u_{0 i}=\left(\begin{array}{c}
\alpha f_{i} \\
d_{i}-C \delta u_{i}
\end{array}\right)
$$

Initially, we set $\delta u_{i}=0$ (no scattered wavefields). After obtaining $u_{0}$ from equation 12 , the secondary source can be updated using $s_{i}=-\omega^{2} \delta m u_{0 i}$. We solve the linear equation 8 to get a new scattered wavefield $\delta u_{i}$. Then the calculated $\delta u_{i}$ is used in equation 12 again to remove the scattered components in the reconstructed background wavefield $u_{0}$, and the secondary source $s_{i}$ will also be updated.

Now, all the ingredients for REWI given by equation 7 are described. Specifically, we first solve equation 8 with $s_{e i}=s_{i}$, then we update the modified secondary source function using $s_{e i}=L_{0} \delta u_{i}$. We use cheap inner iterations to calculate $\delta u_{i}$ and $s_{e i}$ to include multiscattering components in the reconstructed scattered wavefield (Alkhalifah and Song, 2019). We use two inner iterations in all the examples shown in this paper, as multiscattering energy higher than the second order is usually weak. Similar to how we calculate the velocity perturbation in the original EWI, the background model perturbation is evaluated as:

$$
\delta m_{0}=\Re\left(\sum_{i} \frac{\left(s_{i}-s_{e i}\right) \delta u_{i}^{*}}{\omega^{2} \delta u_{i} \delta u_{i}^{*}+\lambda}\right) .
$$


Finally, the background model can be updated using:

$$
m_{0}^{(k+1)}=m_{0}^{(k)}+\delta m_{0},
$$

where $k$ indicates the iteration number. At each frequency, we update the background model once, and we use outer iterations, sweeping the full frequency band, to refine the inverted background model (Song and Alkhalifah, 2020). We outline the background model inversion part in Algorithm 2. To quantitatively demonstrate the performance of different methods, the percent root-mean-square (RMS) errors of the inverted velocity models are evaluated by:

$$
R M S=\frac{1}{\sqrt{N}}\left\|\frac{m_{\text {inv }}-m_{\text {true }}}{m_{\text {true }}}\right\|,
$$

where $N$ is the total number of grid points.

\section{NUMERICAL EXAMPLES}

In this section, we apply our proposed method on two synthetic examples and a real data set to demonstrate its effectiveness. In all the implementations of EWI and REWI shown in this paper, we use two inner iterations at each frequency to generate the inverted velocity models.

\section{Sigsbee2A model}

We first apply the proposed method on a small section of the Sigsbee2A model. The true velocity model is shown in Figure 1a, and the initial velocity is linearly increasing with depth, as shown in Figure 1b. The size of the model is $465 \times 143$, and the spatial sampling interval in both vertical and horizontal directions is $16 \mathrm{~m}$. We use a $8 \mathrm{~Hz}$ Ricker wavelet, which is transformed into the frequency domain to generate the synthetic data. We use the 
same propagator for generating the synthetic data and performing the velocity inversion.

We use 23 sources uniformly distributed on the surface with a horizontal interval of 320 $\mathrm{m}$, and the data are recorded at a depth of $16 \mathrm{~m}$ using 465 receivers. We implement the inversion in the frequency domain, and the selected frequency band used in this example extends from $5 \mathrm{~Hz}$ to $10 \mathrm{~Hz}$ with a sampling interval of $0.5 \mathrm{~Hz}$. The $\alpha^{2}$ and $\epsilon^{2}$ used in all examples in this paper are $10^{7}$ and $10^{12}$, respectively. We select the two weighting factors based on trial and error. We use ten outer iterations to perform REWI, and the inverted perturbation $\delta m$ is shown in Figure 2. After calculating $\delta m$, the background squared slowness update $\delta m_{0}$ at $5 \mathrm{~Hz}$ using REWI is shown in Figure 3a. We can see that low-wavenumber updates are obtained in the shallow part of the model. We also show the squared slowness updates of FWI and EWI in Figure 3b and 3c, respectively. It is obvious that raw velocity gradients of FWI and EWI contain high-wavenumber updates, especially in the circled areas, which may lead to a wrong update direction, considering the poor background model. After ten outer iterations, the inverted background model is shown in Figure 4a, and we observe that we recover a reasonably good smooth background model. The inverted perturbation corresponding to the inverted background model is shown in Figure $4 \mathrm{~b}$, and the reflectors are better focused as the red arrow points out. We also show angle-domain common-image gathers (ADCIGs) at locations $x=\{2,3,4,5,6\} \mathrm{km}$ from $0^{\circ}$ to $60^{\circ}$ obtained using the space-shift imaging condition (Sava and Fomel, 2003). We observe improvements in ADCIGs flatness in multiple areas in Figure 5, which indicates a large improvement in the kinematics of the inverted model.

To introduce high-resolution components into the final inverted velocity model, we implement a sequential FWI and EWI using the inverted background velocity model as the initial model. After ten outer iterations sweeping from $5 \mathrm{~Hz}$ to $10 \mathrm{~Hz}$ with a frequency 
sampling of $0.5 \mathrm{~Hz}$, the final inverted models from the sequential FWI and EWI are shown in Figure 6a and 6b, respectively. We apply a total variation (TV) denoising to the inverted models at each frequency to preserve the sharp edges of the structures (Song and Alkhalifah, 2020). It is obvious that the final inverted models from the sequential FWI and EWI have reasonably recovered most of the true model. With the initial velocity model in Figure 1b, and for comparison, we perform the conventional FWI and the original EWI using the same inversion setup, and the inverted models are shown in Figure $6 \mathrm{c}$ and 6d, respectively. Even though EWI performs better than FWI, they both clearly suffer from the cycle-skipping issue to varying degrees, especially in the circled area. That is because we start the inversion from a relatively high frequency, which is considered a big challenge for FWI. We show in Figure 7 the percent RMS errors in the inverted velocity models per iteration measured with respect to the true model. In Figure 7, iteration zero indicates the RMS errors of starting models for different methods. Both FWI and EWI use the initial velocity model shown in Figure 1b, while a sequential FWI and EWI start with the REWI inverted velocity model shown in Figure 4a (with a lower RMS error). It is obvious that FWI converges to the wrong model due to cycle skipping. The RMS errors for the model inverted with EWI are lower than those obtained using FWI; starting from the REWI inverted model, FWI and EWI (especially EWI) achieve better convergence than using FWI and EWI directly. For details, we compare vertical profiles of the inverted velocity models with the true ones for locations $3.2 \mathrm{~km}$ and $4.8 \mathrm{~km}$, as shown in Figure 8a and 8b, respectively. They both show that REWI is able to recover the smooth velocity in the shallow area, and the sequential FWI and EWI inverted velocity models match the true velocity model better than direct FWI and EWI applications. As the difference in the velocity model between the sequential FWI and EWI is small, we do not show the inverted velocity model using the sequential 
FWI. To summarize, it is important to implement REWI to obtain an accurate background model without low-frequency information.

\section{Volve OBC data}

We finally apply the proposed method to a 2D line extracted from the 3D Volve OBC field data set, which was acquired in the Norwegian North Sea. The survey area is characterized by a small dome-shaped structure (Szydlik et al., 2007). Twelve parallel cables with a sampling interval of $400 \mathrm{~m}$ were used to record the data. Each cable contains 240 receivers, which are evenly distributed in the middle of the survey area every $25 \mathrm{~m}$; the average depth of the receivers is $90 \mathrm{~m}$. The provided tomography-based $\mathrm{P}$-wave velocity model is shown in Figure 9, which was built using well-log information. In the velocity model obtained using tomography, we observe a chalk layer at a depth between $2.5 \mathrm{~km}$ and $3.0 \mathrm{~km}$, and there is a low velocity zone right beneath it, corresponding to the reservoir area. We are provided with preprocessed (denoising and refraction muting) PP and PS data, and we use here the PP data only to perform the proposed method. We use 121 shot gathers in our test, and the horizontal spacing between shots is $100 \mathrm{~m}$ at a depth of $6 \mathrm{~m}$. The maximum offset is almost equal to $5 \mathrm{~km}$. We show one shot gather (PP component) located in the middle of survey area in Figure 10. We observe that the far-offset direct arrivals and the diving waves are muted: we will have to rely on reflections only. We extract the source wavelet by back propagating the near-offset direct waves to the source location (Plessix, 2006). The reflections in the data are clean, so we mainly use them to invert for the background model. We choose the 61st trace of the displayed shot gather (in Figure 10) and analyse the frequency content of the recorded data. The original trace and the frequency spectrum corresponding to it are shown in Figure 11a and 11b, respectively. We apply a bandpass 
filter between $3 \mathrm{~Hz}$ and $10 \mathrm{~Hz}$. The filtered data frequency spectrum and data are shown in Figure 11c and 11d, respectively. We observe that the low-frequency components in the data are missing. We smooth the provided tomography velocity three times with a Gaussian window size of $500 \times 500 \mathrm{~m}$ and use it as the initial background model for the inversion, as shown in Figure 12.

In this field data test, the frequency band used starts from $3 \mathrm{~Hz}$ to $10 \mathrm{~Hz}$ with a 0.3 sampling interval. We first perform FWI and EWI using ten iterations for each frequency with a source estimation step (Pratt, 1999; Fang et al., 2018). In this velocity inversion, since the initial velocity is generally good for the shallow area, we do not update the background velocity above z: $1 \mathrm{~km}$. The inverted velocity models using FWI and EWI are shown in Figure 13a and 13b, respectively. As the real data lack low-frequency components and diving waves, both FWI and EWI fail to recover the chalk layer above the reservoir between z: $2.5 \mathrm{~km}$ and z: $3.0 \mathrm{~km}$.

Then, we perform ten outer iterations sweeping all the selected frequencies to invert for the background velocity model using REWI. Using the initial velocity in Figure 12, the inverted perturbation model is shown in Figure 14. We note layered structures in the perturbation model. After ten outer iterations, the inverted background model is shown in Figure 15, and we see that the chalk layer and a low velocity zone, at the reservoir layer, between $2.5 \mathrm{~km}$ and $3.0 \mathrm{~km}$ is recovered. Figure 16 shows the model perturbation corresponding to the inverted background velocity. As the background model improves, the structural information can be seen more clearly in the perturbation model, especially in the reservoir area. We calculate ADCIGs at $x=\{4,5,6,7,8\} \mathrm{km}$ ranging between $0^{\circ}$ to $40^{\circ}$ for the initial and inverted velocity models, as shown in Figure 17a and 17b, respectively. In Figure 17a, we observe the ADCIGs corresponding to the initial velocity are not flat 
in multiple areas, which indicates the poor quality of the initial model. Such an initial velocity may cause serious cycle-skipping issues for FWI, and even EWI. In addition, only reflected waves are available in the data, which may pose additional challenges for FWI and EWI. By comparison, the ADCIGs corresponding to the inverted velocity (in Figure 17b) are reasonably flat in multiple areas (as the red arrows point out) compared to those corresponding to the initial velocity. This indicates an improvement in the inverted velocity in the deep areas. This improvement is due to the key feature (accurate estimation of the background velocity model) in REWI, which inherently uses reflected waves in the inversion.

Because it is based on the $L_{2}$ norm of the data residual, reflection-based waveform inversion method tends to focus on the strongest reflectors; it often has poor vertical resolution as well. To refine the inverted background velocity model, we use EWI to perform a sequential inversion with all the selected frequencies. After five iterations, the final inverted model is shown in Figure 18, and we notice some details added to the inverted model. In addition, we compare the inverted velocity with one well-log profile from a check shot nearby, and we observe that the inverted velocity agrees well with the check-shot profile, as shown in Figure 19. By comparison, FWI and EWI inverted velocity models fail to capture the high velocity of the chalk layer. In addition, FWI generates unreasonable high velocity updates in the deep area at a $4 \mathrm{~km}$ depth. Thus, we do not show FWI and EWI inverted velocity models in the velocity profiles. Finally, we compare the true shot gather (bandpass of 2-6 $\mathrm{Hz})$ at $6.175 \mathrm{~km}$ with the data generated by the initial velocity and the inverted velocity using a time-domain acoustic wave equation propagator with a free-surface boundary condition. In Figure 20a, we can see that many reflections are missing in the predicted data using the initial velocity model. By comparison, the reflections in the predicted data are recovered using the inverted velocity, and they match the recorded data reasonably well, 
as shown in Figure 20b. We use a dashed red line in Figure 20b to show the data fitting at the far offsets. We also compare the shot gather at $5.175 \mathrm{~km}$ with the predicted data corresponding to the initial and inverted velocity models, as shown in Figure 21a and 21b, respectively. We come to the conclusion that the inverted velocity can recover the reflections present in the recorded data. We observe slight mismatches between the observed and predicted data due to the presence of anisotropy in the survey are that is not taken into account in our wave equation propagator (Guitton and Alkhalifah, 2017; Oh et al., 2018).

\section{DISCUSSION}

We use a reflection-based efficient wavefield inversion (REWI) method to invert for the background model. It provides an alternative way to LSRTM of calculating the perturbation model efficiently. Since perturbations in the subsurface are considered as secondary sources generating the scattered wavefield, we can estimate these perturbations when the scattered wavefield is reconstructed. This process is theoretically equivalent to the estimation of the microseismic events in the subsurface (Song and Alkhalifah, 2019). The calculation of the perturbations is implemented in each outer iteration prior to the background model inversion step. When increasing the number of outer iterations, the background model matures. As a result, the structures in the perturbation model gets better focused and more continuous after each outer iteration. At the early outer iterations, the structures may be poorly positioned due to kinematic errors in the background model, and this will cause mismatches in the reflection data. REWI is able to overcome this issue by using the Born approximation of the wave equation for the Born scattering as a regularization term.

The main difference between the original EWI and the proposed REWI is in the way that the reflected events are used. In conventional FWI and EWI, the reflections are mainly 
used to invert for their scattering components, with little update to the wave path (or wave path velocity) in the early iterations. As a result, with a poor initial velocity, reflections are the main cause of cycle skipping, which will lead the convergence inversion to a local minimum. Although EWI reduces the cycle-skipping issue compared to FWI, by allowing for more data fitting, this feature has a limit (Alkhalifah and Song, 2019). To overcome these challenges, conventional RWI and the proposed REWI inverts for the reflectivity and use it to update both the wavefield and the velocity along the reflection wavepaths. Compared to the conventional RWI, REWI improves the efficiency of the reflectivity calculation and enhances matching of the reflection data.

The weighting factors $\alpha^{2}$ and $\epsilon^{2}$ for EWI and REWI, respectively, are important for mitigating cycle skipping. A large $\alpha^{2}$ or $\epsilon^{2}$ means that little data information is used to reconstruct the wavefield. On the other hand, a relatively small $\alpha^{2}$ or $\epsilon^{2}$ means more data fitting is allowed. Thus, the cycle-skipping problem can be somewhat mitigated (Alkhalifah and Song, 2019). We use trial and error to find the appropriate weighting factors. A quick way to find a proper $\alpha^{2}$ value is set it to a portion of the highest eigenvalue of the auxiliary linear system (van Leeuwen and Herrmann, 2015). We can also use a multiplicative cost function, in which $\alpha^{2}$ can be automatically determined (da Silva and Yao, 2017).

Either in the perturbation or background model inversion steps, we use a band of frequencies to perform the inversion. This is because monofrequency waveform inversion cannot provide continuous wavenumber coverage of the perturbation and background models (Sirgue and Pratt, 2004). To reduce aliasing effects, multiple frequencies are often used in frequency-domain waveform inversion, even with a narrow frequency band. The proposed method is mainly efficient in the frequency domain due to the LU decomposition, as EWI requires inner iterations to include multiscattering components in the reconstructed wave- 
field. However, frequency-domain waveform inversion methods require significant storage and have a high computational cost, especially for industrial-size 3D models (Pratt, 1999; Wang et al., 2011). The approach also faces, like with any frequency domain inversion, challenges when dealing with anisotropy and elasticity, as it requires the calculation of a larger stiffness matrix (Lee et al., 2010; Li et al., 2018). A dual formulation for time-domain wavefield reconstruction inversion was proposed, and it requires twice the computational cost of FWI (Rizzuti et al., 2019). As a result, the proposed method is possible, but expensive, in a time-domain implementation.

\section{CONCLUSION}

We developed a reflection-based efficient wavefield inversion method, called REWI, to perform the inversion of a background velocity inversion using data without low frequencies. We calculate the perturbation model in an efficient manner before the inversion of the background model starts. The perturbation may be located initially at the wrong depth, which often results in reflection data mismatch for conventional RWI. In REWI, we use the wave equation for the Born scattered wavefield as a regularization term, instead of a constraint, which allows for more reflection data matching resulting in less cycle-skipping in reflection waveform inversion. Inner iterations between the scattered wavefield reconstruction and the update of the modified secondary source allow the multiscattering components to contribute to the background model update as well. We update the background model once per frequency, and use the outer iterations to refine it. Finally, a sequential EWI is used to introduce the detailed structures of the velocity model. Applications on synthetic and field datasets demonstrate that REWI can recover a reasonably good background model. 


\section{ACKNOWLEDGMENTS}

The authors would like to thank Statoil ASA and the Volve license partners ExxonMobil E\&P Norway AS and Bayerngas Norge AS, for the release of the Volve data. The views expressed in this paper are the views of the authors and do not necessarily reflect the views of Statoil ASA and the Volve field license partners. We thank the King Abdullah University of Science and Technology (KAUST) for sponsoring this research. We thank our Seismic Wave Analysis Group colleagues for their helpful suggestions. We also thank the editor Dr.Jeffrey Shragge, the associate editor Dr.Anatoly Baumstein, the assistant editor Dr. Antoine Guitton, and six anonymous reviewers for their important suggestions and comments. 


\section{REFERENCES}

Alkhalifah, T., and C. Song, 2019, An efficient wavefield inversion: Using a modified source function in the wave equation: Geophysics, 84, no.6, R909-R922.

Alkhalifah, T., and Z. Wu, 2016, The natural combination of full and image-based waveform inversion: Geophysical Prospecting, 64, 19-30.

Biondi, B., and A. Almomin, 2014, Simultaneous inversion of full data bandwidth by tomographic full-waveform inversion: Geophysics, 79, no.3, WA129-WA140.

Biondi, B., and W. W. Symes, 2004, Angle-domain common-image gathers for migration velocity analysis by wavefield-continuation imaging: Geophysics, 69, no.5, 1283-1298.

Bozdăg, E., J. Trampert, and J. Tromp, 2011, Misfit functions for full waveform inversion based on instantaneous phase and envelope measurements: Geophysical Journal International, $\mathbf{1 8 5}, 845-870$.

Chi, B., L. Dong, and Y. Liu, 2014, Full waveform inversion method using envelope objective function without low frequency data: Journal of Applied Geophysics, 109, 36-46.

Choi, Y., and T. Alkhalifah, 2013, Frequency-domain waveform inversion using the phase derivative: Geophysical Journal International, 195, 1904-1916.

Clement, F., G. Chavent, and S. Gómez, 2001, Migration-based traveltime waveform inversion of 2-d simple structures: A synthetic example: Geophysics, 66, no.3, 845-860.

da Silva, N. V., and G. Yao, 2017, Wavefield reconstruction inversion with a multiplicative cost function: Inverse Problems, 34, 015004.

Fang, Z., R. Wang, and F. J. Herrmann, 2018, Source estimation for wavefieldreconstruction inversion: Geophysics, 83, no.4, R345-R359.

Guitton, A., and T. Alkhalifah, 2017, A parameterization study for elastic VTI fullwaveform inversion of hydrophone components: Synthetic and North Sea field data ex- 
amples: Geophysics, 82, no.6, R299-R308.

Lee, H.-Y., J. M. Koo, D.-J. Min, B.-D. Kwon, and H. S. Yoo, 2010, Frequency-domain elastic full waveform inversion for VTI media: Geophysical Journal International, 183, 884-904.

Li, J., K. A. Innanen, W. Pan, and G. Yu, 2018, Frequency domain elastic FWI for VTI media: 88th Annual International Meeting, SEG, Expanded Abstracts, 1223-1227.

Li, Y., and T. Alkhalifah, 2020, Multi-parameter reflection waveform inversion for acoustic transversely isotropic media with a vertical symmetry axis: Geophysical Prospecting, 68, $1878-1892$.

Li, Y. E., and L. Demanet, 2016, Full-waveform inversion with extrapolated low-frequency data: Geophysics, 81, no.6, R339-R348.

Liu, D.-J., J.-P. Huang, and Z.-Y. Wang, 2020, Convolution-based multi-scale envelope inversion: Petroleum Science, 17, 1-11.

Mora, P., 1989, Inversion=migration+tomography: Geophysics, 54, no.12, 1575-1586.

Oh, J.-W., M. Kalita, and T. Alkhalifah, 2018, 3D elastic full-waveform inversion using P-wave excitation amplitude: Application to ocean bottom cable field data: Geophysics, 83, no.2, R129-R140.

Ovcharenko, O., V. Kazei, M. Kalita, D. Peter, and T. Alkhalifah, 2019, Deep learning for low-frequency extrapolation from multioffset seismic data: Geophysics, 84, no.6, R989R1001.

Plessix, R.-E., 2006, A review of the adjoint-state method for computing the gradient of a functional with geophysical applications: Geophysical Journal International, 167, 495503.

Pratt, R. G., 1999, Seismic waveform inversion in the frequency domain; part 1, theory and 
verification in a physical scale model: Geophysics, 64, no.3, 888-901.

Rizzuti, G., M. Louboutin, R. Wang, E. Daskalakis, and F. Herrmann, 2019, A dual formulation for time-domain wavefield reconstruction inversion: 89th Annual International Meeting, SEG, Expanded Abstracts, 1480-1485.

Sava, P. C., and S. Fomel, 2003, Angle-domain common-image gathers by wavefield continuation methods: Geophysics, 68, no.3, 1065-1074.

Shin, C., and Y. H. Cha, 2008, Waveform inversion in the laplace domain: Geophysical Journal International, 173, 922-931.

Sirgue, L., and R. G. Pratt, 2004, Efficient waveform inversion and imaging: A strategy for selecting temporal frequencies: Geophysics, 69, no.1, 231-248.

Song, C., and T. Alkhalifah, 2019, Microseismic event estimation based on an efficient wavefield inversion: IEEE Journal of Selected Topics in Applied Earth Observations and Remote Sensing, 12, 4664-4671.

— 2020, Efficient wavefield inversion with outer iterations and total variation constraint: Transactions on Geoscience and Remote Sensing, 58, 5836-5846.

Sun, D., K. Jiao, X. Cheng, Z. Xu, L. Zhang, and D. Vigh, 2017, Born modeling based adjustive reflection full waveform inversion: 87th Annual International Meeting, SEG, Expanded Abstracts, 1460-1465.

Symes, W. W., 2008, Migration velocity analysis and waveform inversion: Geophysical prospecting, 56, 765-790.

Szydlik, T., P. Smith, S. Way, L. Aamodt, and C. Friedrich, 2007, 3D PP/PS prestack depth migration on the Volve field: First break, 25, 43-47.

Tarantola, A., 1984, Inversion of seismic reflection data in the acoustic approximation: Geophysics, 49, no.8, 1259-1266. 
Van Leeuwen, T., and F. J. Herrmann, 2013, Mitigating local minima in full-waveform inversion by expanding the search space: Geophysical Journal International, 195, 661667.

, 2015, A penalty method for pde-constrained optimization in inverse problems: Inverse Problems, 32, 015007.

Wang, G., S. Wang, Q. Du, and S. Yuan, 2017, Traveltime-based reflection full-waveform inversion for elastic medium: Journal of Applied Geophysics, 141, 68-76.

Wang, P., Z. Zhang, Z. Wei, and R. Huang, 2018, A demigration-based reflection fullwaveform inversion workflow: 88th Annual International Meeting, SEG, Expanded Abstracts, $1138-1142$.

Wang, S., M. V. de Hoop, and J. Xia, 2011, On 3D modeling of seismic wave propagation via a structured parallel multifrontal direct Helmholtz solver: Geophysical Prospecting, $\mathbf{5 9}, 857-873$.

Wu, R.-S., J. Luo, and B. Wu, 2014, Seismic envelope inversion and modulation signal model: Geophysics, 79, no.3, WA13-WA24.

Wu, Z., and T. Alkhalifah, 2015, Simultaneous inversion of the background velocity and the perturbation in full-waveform inversion: Geophysics, 80, no.6, R317-R329.

Xu, S., D. Wang, F. Chen, Y. Zhang, and G. Lambare, 2012, Full waveform inversion for reflected seismic data: 74th Annual International Conference and Exhibition, EAGE, Extended Abstracts, cp-293-00729.

Yao, G., N. V. da Silva, and D. Wu, 2019, Reflection-waveform inversion regularized with structure-oriented smoothing shaping: Pure and Applied Geophysics, 176, 5315-5335.

Yao, G., and D. Wu, 2017, Reflection full waveform inversion: Science China Earth Sciences, 60, 1783-1794. 
Yao, G., D. Wu, and S.-X. Wang, 2020, A review on reflection-waveform inversion: Petroleum Science, 17, 1-18.

Zhang, Y., and L. Duan, 2012, Predicting multiples using a reverse time demigration: 82nd Annual International Meeting, SEG, Expanded Abstracts.

Zhou, H., L. Amundsen, and G. Zhang, 2012, Fundamental issues in full waveform inversion: 82nd Annual International Meeting, SEG, Expanded Abstracts. 


\section{LIST OF FIGURES}

1 (a) The true velocity model extracted from a section of the Sigsbee2A velocity model, and (b) initial velocity.

2 The inverted perturbation corresponding to the initial velocity at the first outer iteration. The arrow points to a poorly focused shallow event.

3 (a) The background squared slowness update using REWI, squared slowness update using (b) FWI and (c) EWI of $5 \mathrm{~Hz}$ at the first iteration. The circled areas show clear wavenumber differences between the velocity updates.

4 (a) The inverted background model after ten outer iterations using REWI, and (b) the inverted perturbation corresponding to the inverted background velocity at the last outer iteration. The arrow points to an improved better focused shallow event compared to Figure 2.

5 ADCIGs at locations $x=\{2,3,4,5,6\} \mathrm{km}$ corresponding to (a) the initial and (b) the REWI inverted velocity models. The arrows point to ADCIGs improvements in multiple areas.

6 The sequential (a) FWI, and (b) EWI inverted velocity models using the REWI inverted background model as the initial velocity model. The (c) FWI, and (d) EWI inverted models using the initial velocity model in Figure 1b. The circled areas show clear differences between the inverted models.

7 The percent RMS error of the inverted velocity models per iteration for the Sigsbee model. Iteration zero indicates the RMS errors for the starting models for different methods.

8 The velocity profiles at locations (a) $x=3.2 \mathrm{~km}$ and (b) $x=4.8 \mathrm{~km}$ in Figure 6.

9 The initial P-wave velocity model obtained by tomography provided with the Volve 
data.

10 One shot gather of the Volve OBC dataset at location $x=6.175 \mathrm{~km}$.

11 (a) The 61st trace of the shot gather in Figure 10, (b) the frequency spectrum of this trace, (c) the filtered trace, and (d) the frequency spectrum after a bandpass filter.

12 The initial velocity model used for the inversion of the background velocity inversion.

13 The (a) FWI and (b) EWI inverted P-wave velocity models.

14 The inverted perturbation corresponding to the initial velocity model at the first outer iteration.

15 The inverted background velocity model using REWI.

16 The inverted perturbation corresponding to the inverted background velocity at the last outer iteration.

17 ADCIGs at $x=\{4,5,6,7,8\} \mathrm{km}$ corresponding to (a) the initial and (b) the REWI inverted velocity models. The arrows point to ADCIGs improvements in multiple areas.

18 The sequential EWI inverted velocity model obtained using the inverted background model as the initial guess.

19 The vertical velocity profiles at location $x=8.24 \mathrm{~km}$ for the various inverted models compared to a check shot velocity profile at the same location.

20 The shot gather at location $x=6.175 \mathrm{~km}$ comparison between the filtered recoded data (left) and the predicted data (right) corresponding to the (a) initial and (b) inverted velocity models. A red dashed line in Figure 20b demonstrates the data fitting the at far offset.

21 The shot gather at location $x=5.175 \mathrm{~km}$ comparison between the filtered recoded data (left) and the predicted data (right) corresponding to the (a) initial and (b) inverted 
velocity models.

This paper presented here as accepted for publication in Geophysics prior to copyediting and composition. (C) 2021 Society of Exploration Geophysicists 

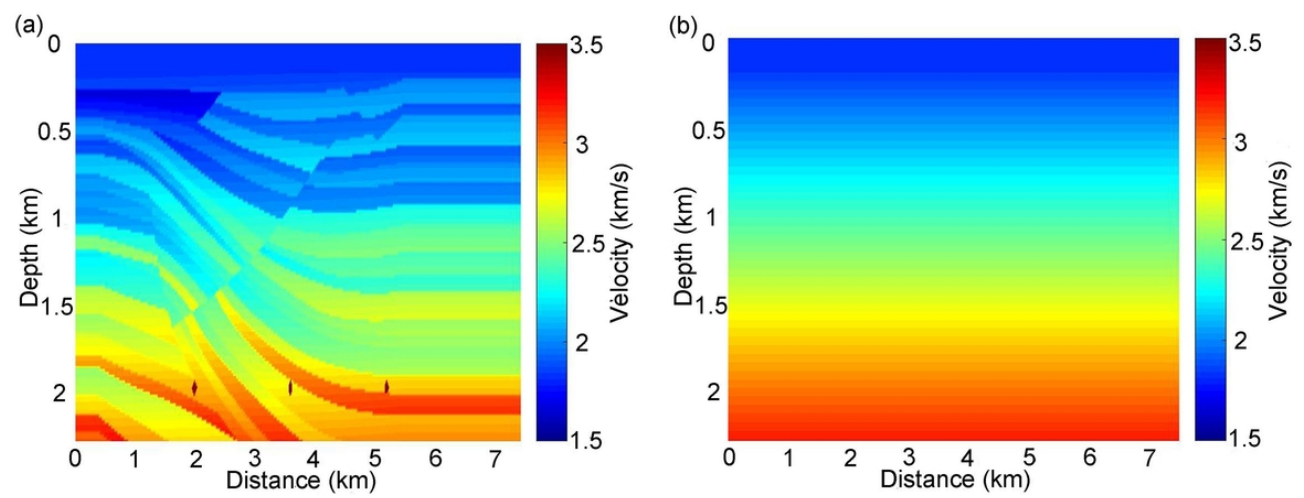

Figure 1: (a) The true velocity model extracted from a section of the Sigsbee2A velocity model, and (b) initial velocity.

$99 \times 37 \mathrm{~mm}(300 \times 300 \mathrm{DPI})$ 


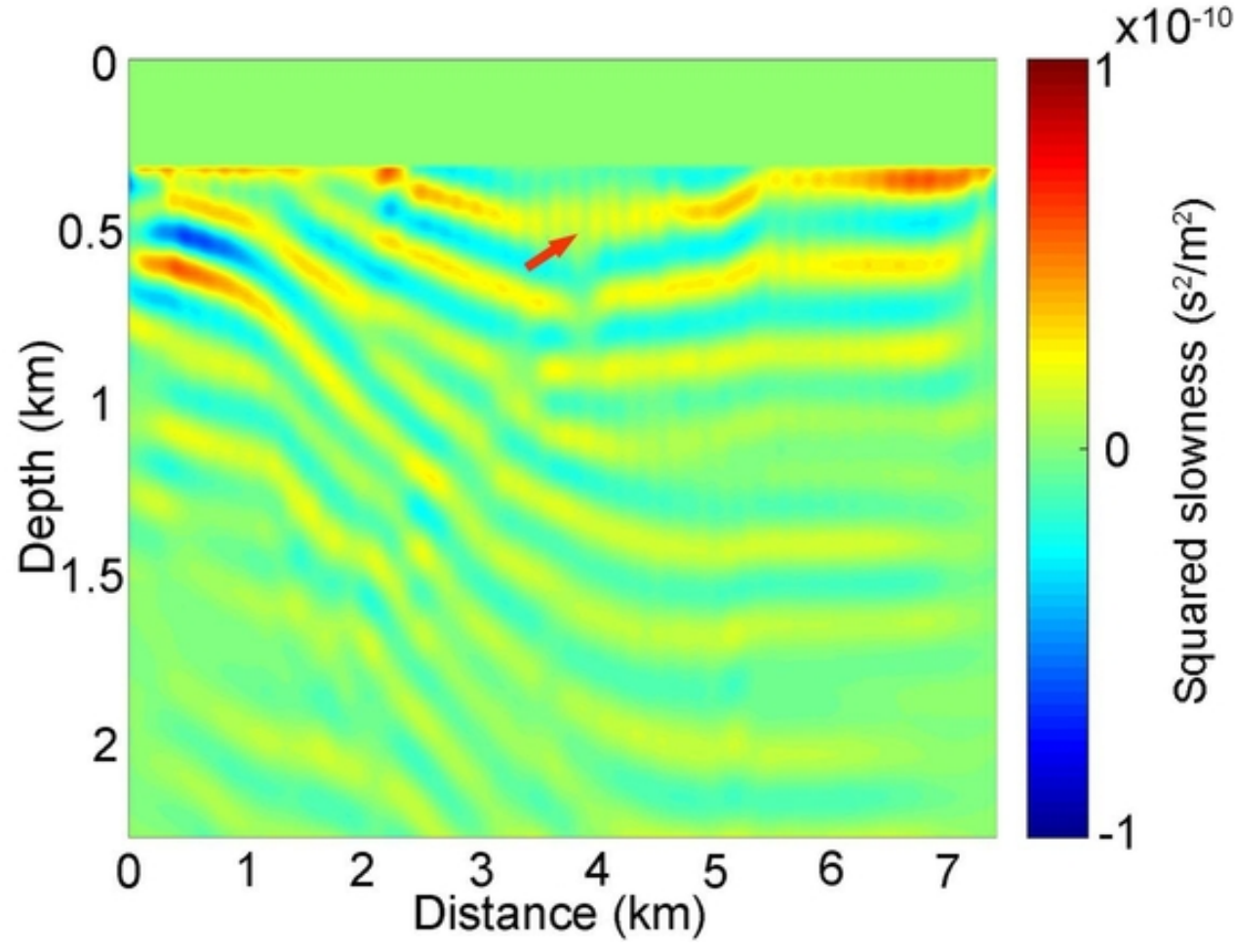

Figure 2: The inverted perturbation corresponding to the initial velocity at the first outer iteration. The arrow points to a poorly focused shallow event.

$$
49 \times 37 \mathrm{~mm}(300 \times 300 \text { DPI })
$$



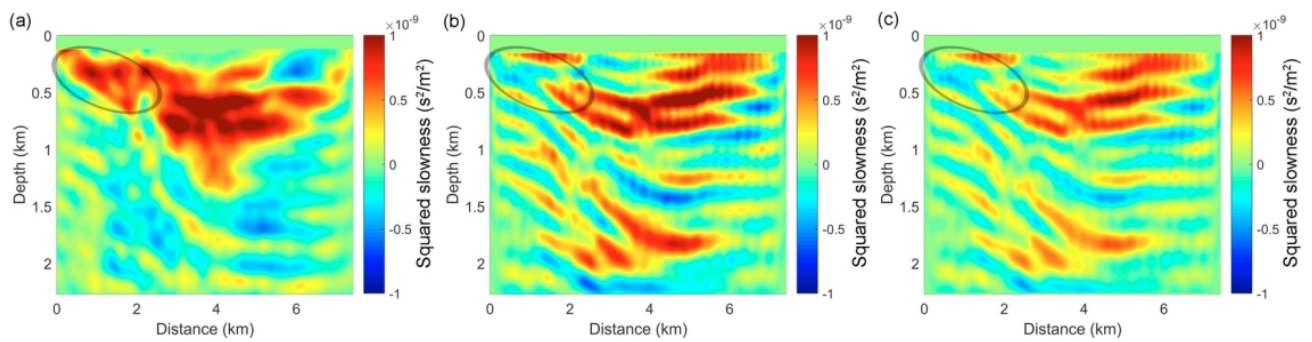

Figure 3: (a) The background squared slowness update using REWI, squared slowness update using (b) FWI and (c) EWI of $5 \mathrm{~Hz}$ at the first iteration. The circled areas show clear wavenumber differences between the velocity updates.

$149 \times 37 \mathrm{~mm}(300 \times 300 \mathrm{DPI})$ 

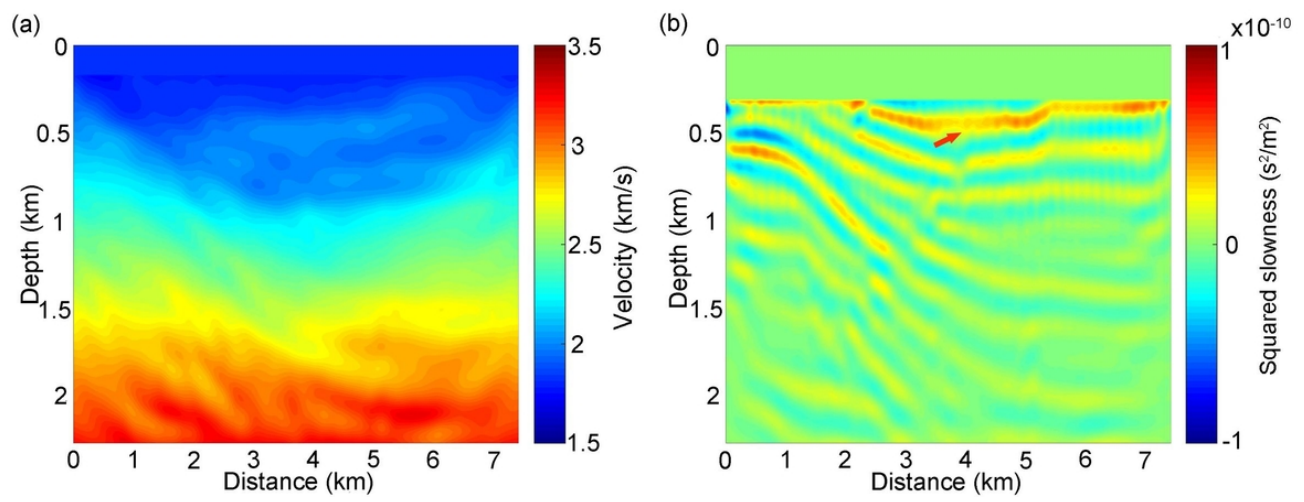

Figure 4: (a) The inverted background model after ten outer iterations using REWI, and (b) the inverted perturbation corresponding to the inverted background velocity at the last outer iteration. The arrow points to an improved better focused shallow event compared to Figure 2.

$$
99 \times 37 \mathrm{~mm}(300 \times 300 \text { DPI })
$$


(a)

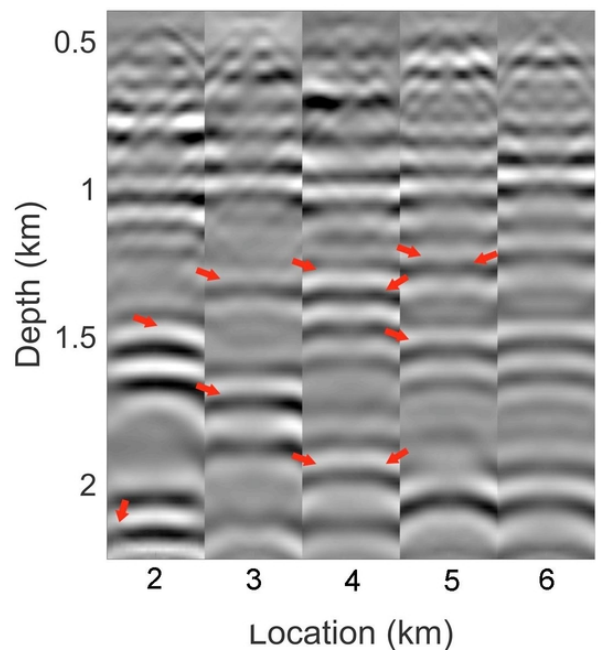

(b)

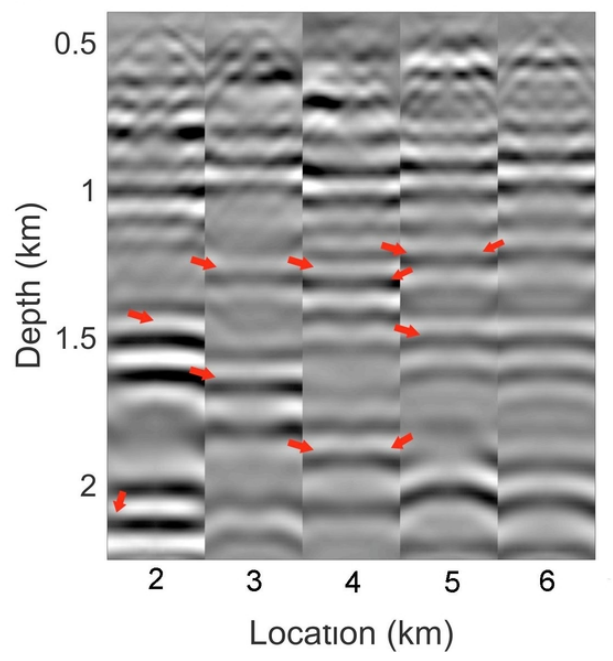

Figure 5: ADCIGs at locations $x=\{2,3,4,5,6\} \mathrm{km}$ corresponding to (a) the initial and (b) the REWI inverted velocity models. The arrows point to ADCIGs improvements in multiple areas.

$99 \times 53 \mathrm{~mm}(300 \times 300 \mathrm{DPI})$ 

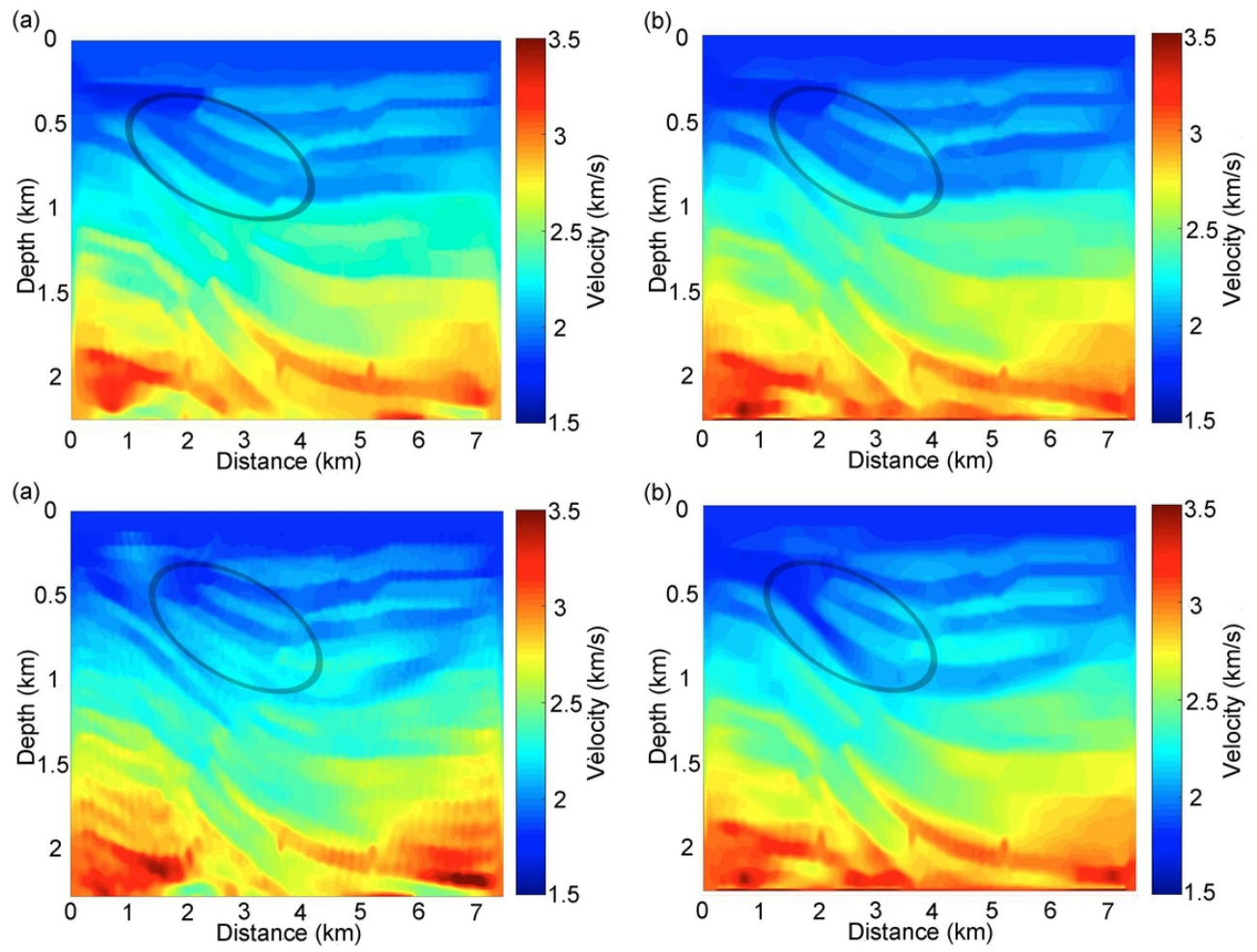

Figure 6: The sequential (a) FWI, and (b) EWI inverted velocity models using the REWI inverted background model as the initial velocity model. The (c) FWI, and (d) EWI inverted models using the initial velocity model in Figure 1b. The circled areas show clear differences between the inverted models.

$99 \times 75 \mathrm{~mm}(300 \times 300 \mathrm{DPI})$ 


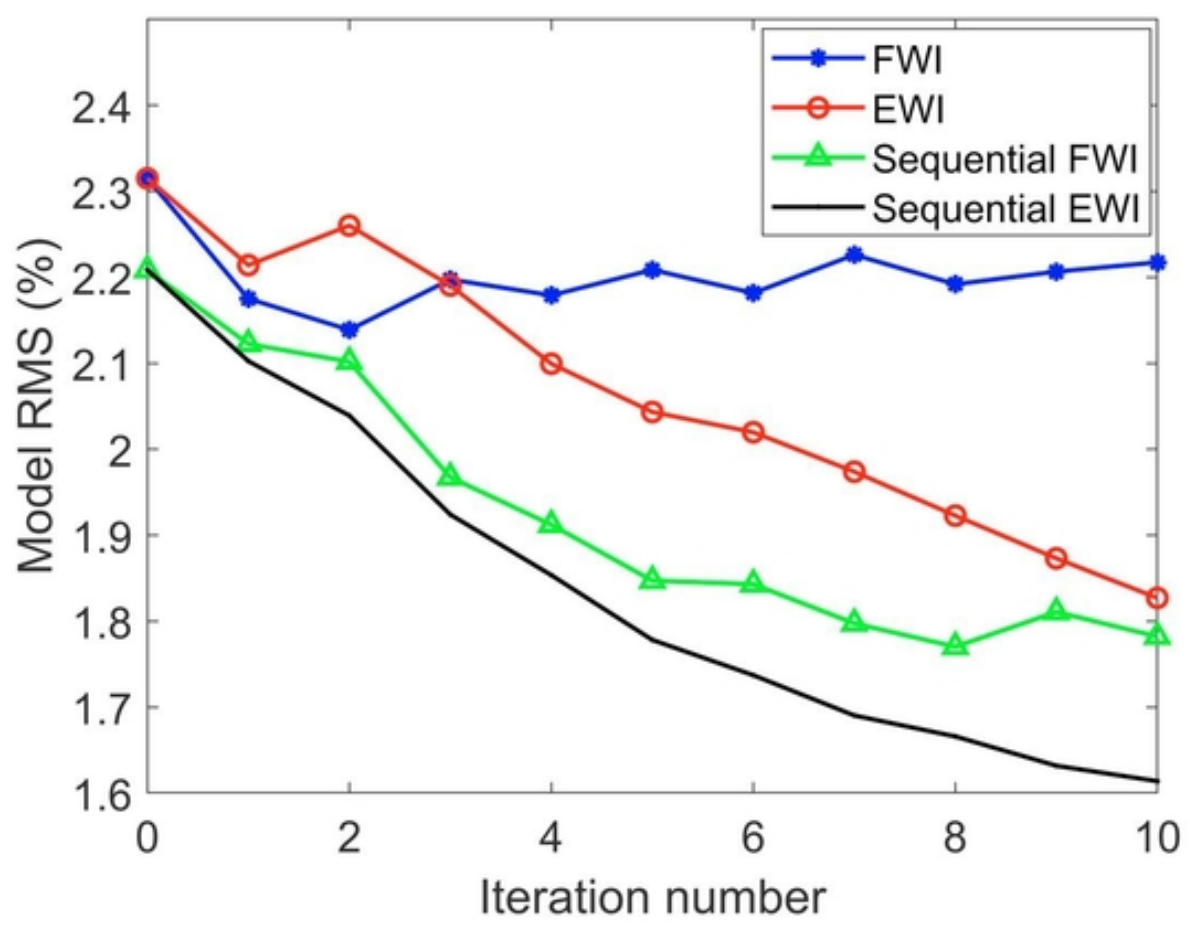

Figure 7: The percent RMS error of the inverted velocity models per iteration for the Sigsbee model. Iteration zero indicates the RMS errors for the starting models for different methods.

$49 \times 37 \mathrm{~mm}(300 \times 300$ DPI $)$ 
(a)

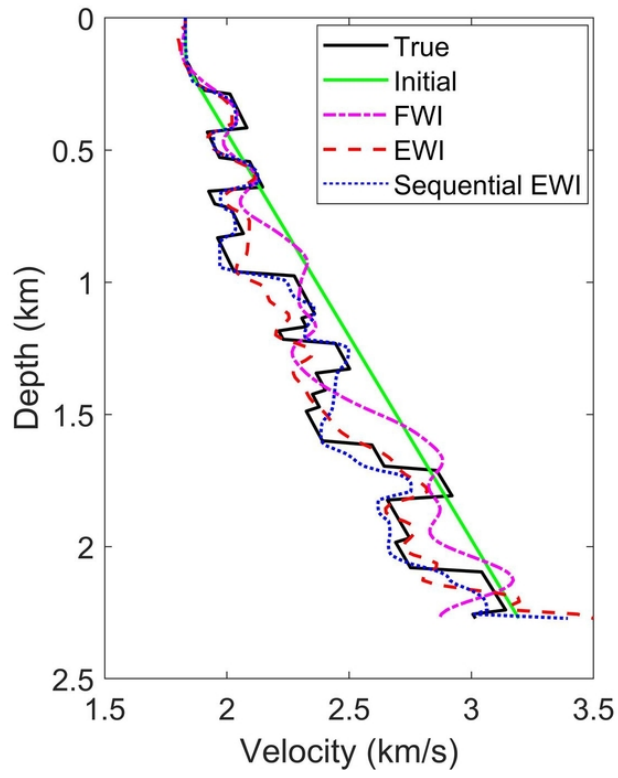

(b)

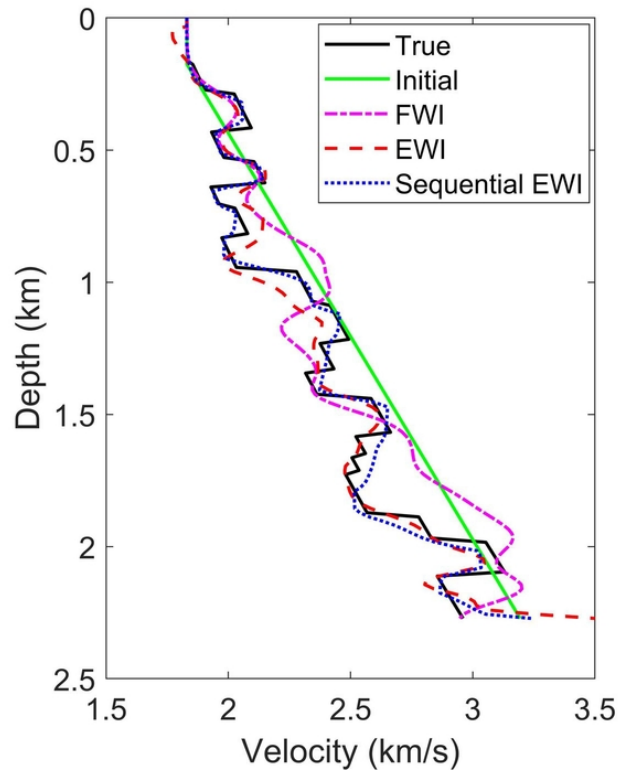

Figure 8: The velocity profiles at locations (a) $x=3.2 \mathrm{~km}$ and (b) $x=4.8 \mathrm{~km}$ in Figure 6 .

$99 \times 62 \mathrm{~mm}(300 \times 300 \mathrm{DPI})$

This paper presented here as accepted for publication in Geophysics prior to copyediting and composition.

(C) 2021 Society of Exploration Geophysicists 


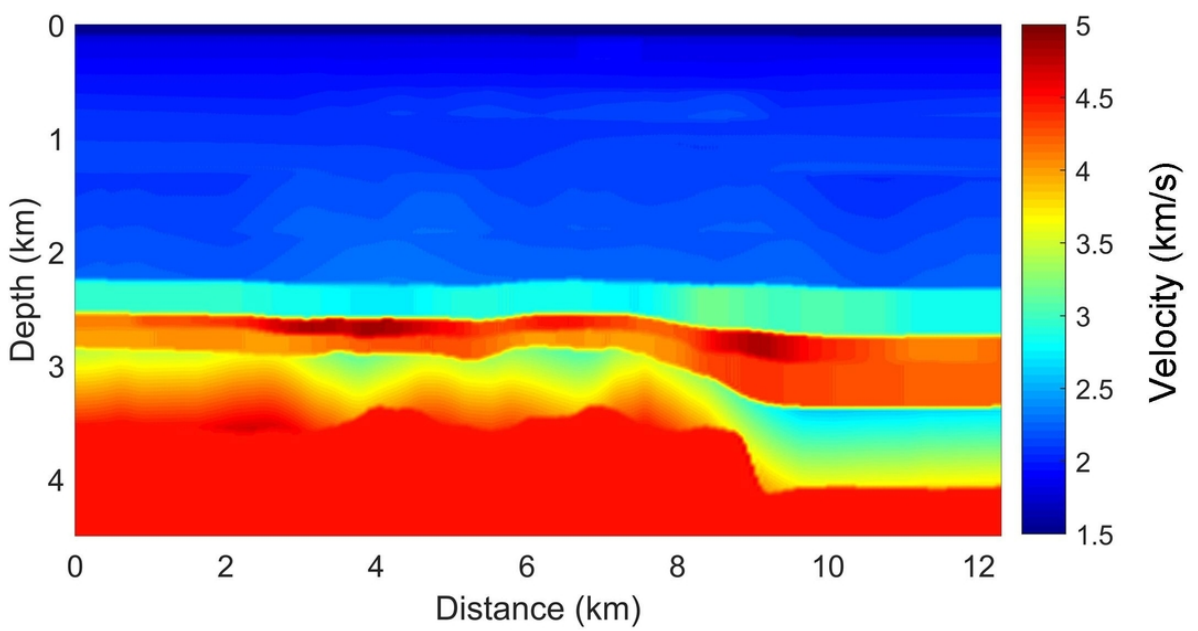

Figure 9: The initial P-wave velocity model obtained by tomography provided with the Volve data.

$$
99 \times 51 \mathrm{~mm}(300 \times 300 \mathrm{DPI})
$$




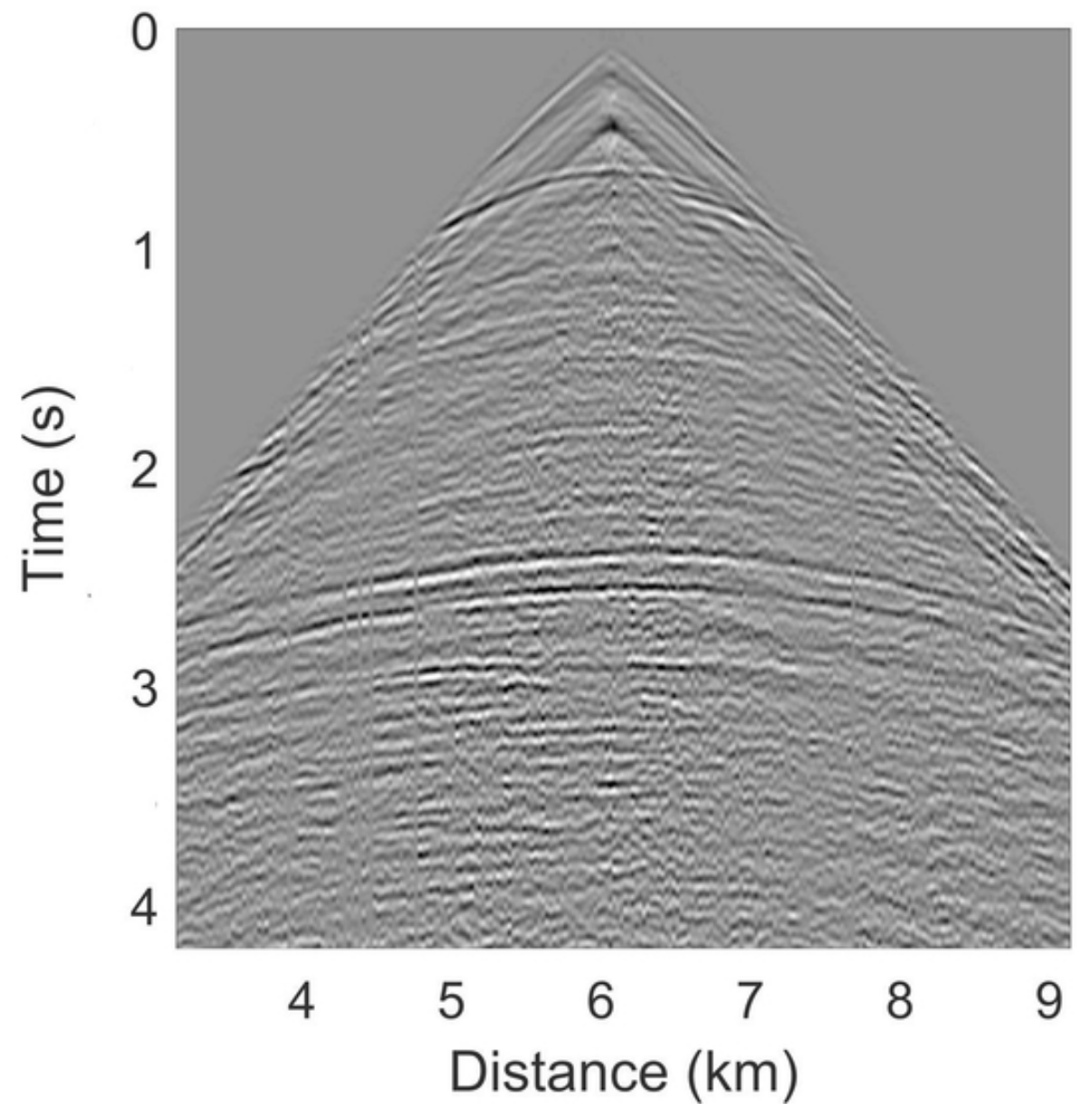

Figure 10: One shot gather of the Volve OBC dataset at location $x=6.175 \mathrm{~km}$.

$49 \times 49 \mathrm{~mm}(300 \times 300 \mathrm{DPI})$

This paper presented here as accepted for publication in Geophysics prior to copyediting and composition.

(C) 2021 Society of Exploration Geophysicists 
(a)

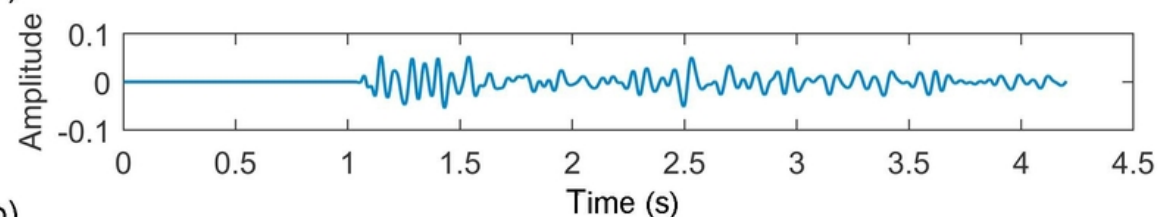

(b)

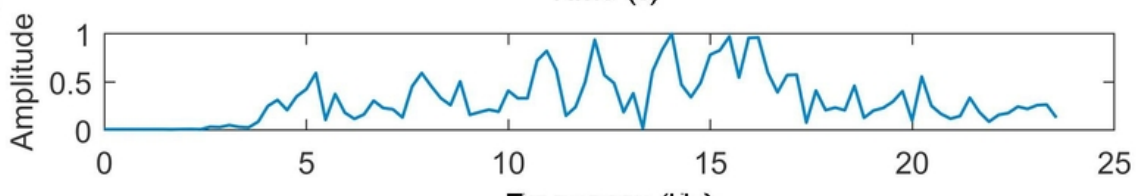

(c)

Frequency $(\mathrm{Hz})$
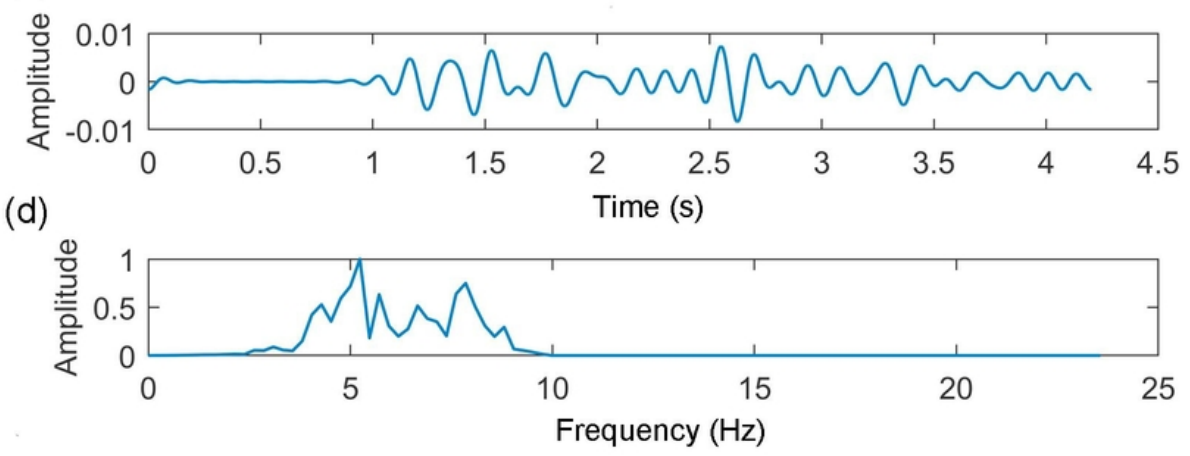

Figure 11: (a) The 61st trace of the shot gather in Figure 10, (b) the frequency spectrum of this trace, (c) the filtered trace, and (d) the frequency spectrum after a bandpass filter.

\section{$75 \times 56 \mathrm{~mm}(300 \times 300$ DPI $)$}

This paper presented here as accepted for publication in Geophysics prior to copyediting and composition.

(C) 2021 Society of Exploration Geophysicists 


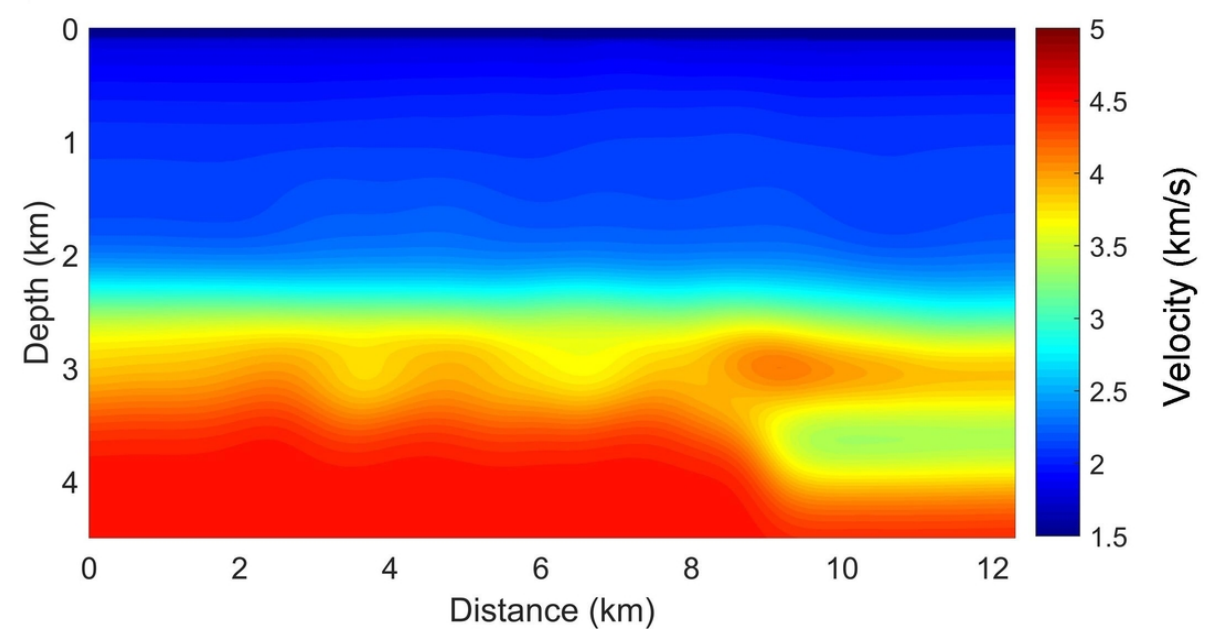

Figure 12: The initial velocity model used for the inversion of the background velocity inversion.

$99 \times 48 \mathrm{~mm}(300 \times 300 \mathrm{DPI})$ 
(a)

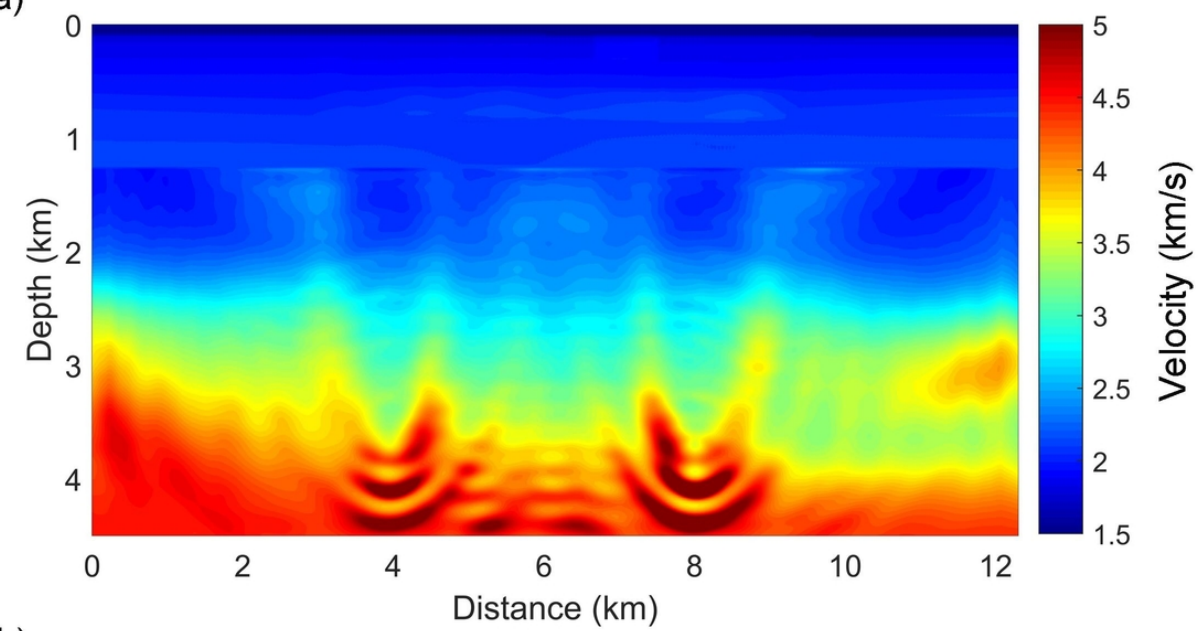

(b)

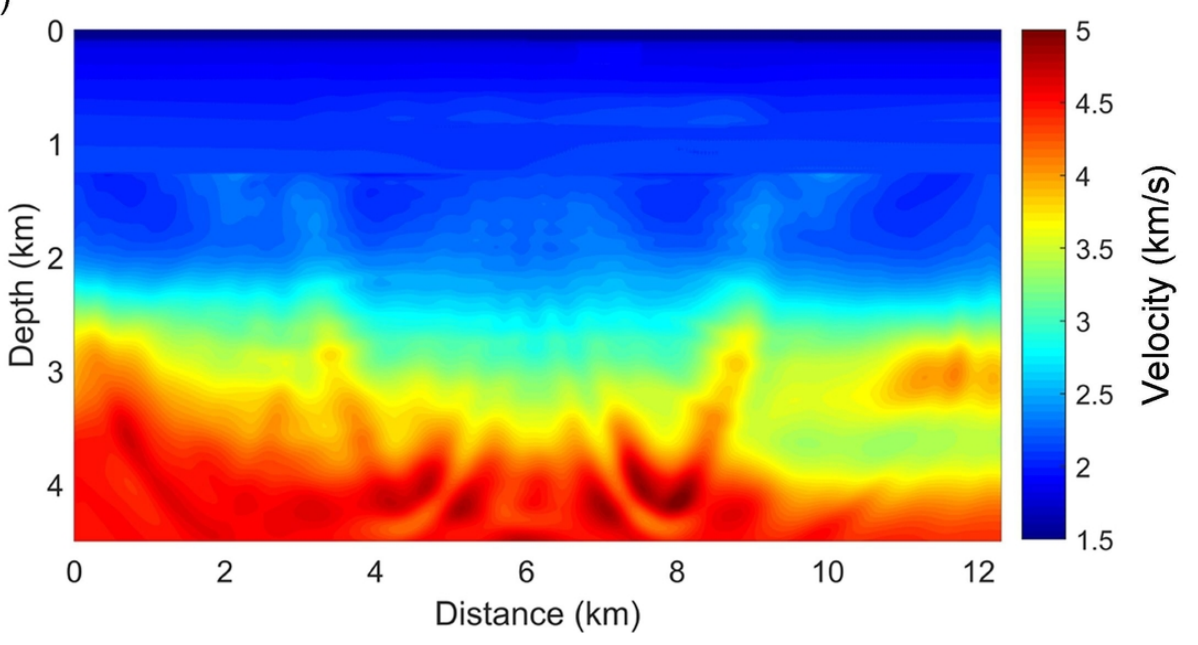

Figure 13: The (a) FWI and (b) EWI inverted P-wave velocity models.

$99 \times 99 \mathrm{~mm}(300 \times 300 \mathrm{DPI})$ 


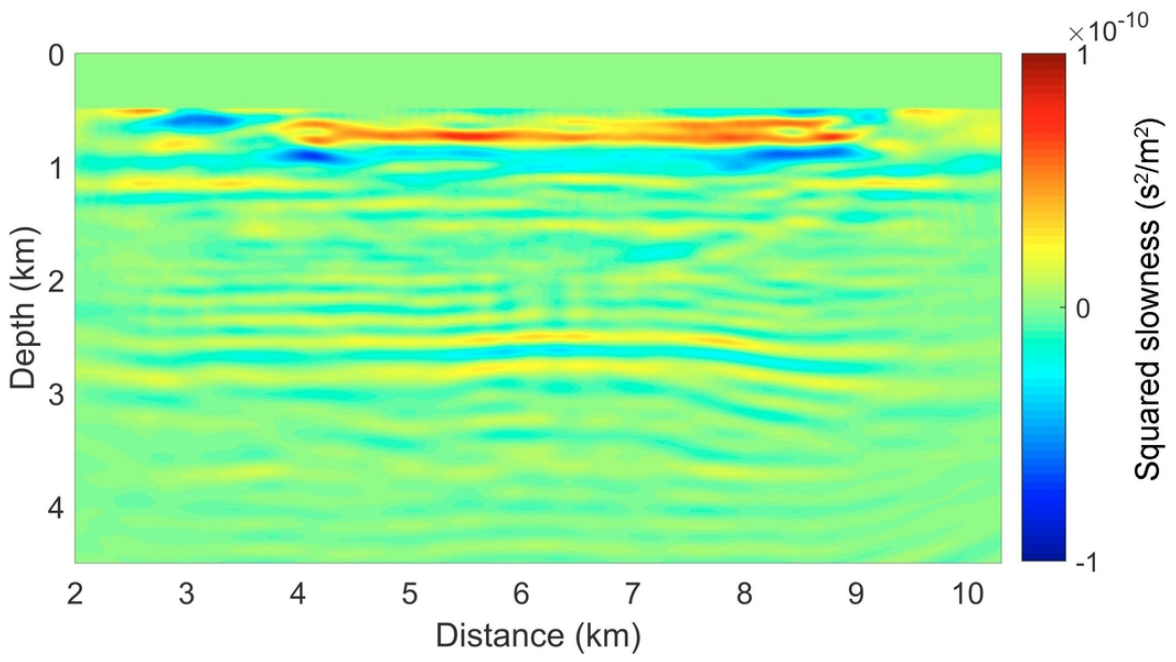

Figure 14: The inverted perturbation corresponding to the initial velocity model at the first outer iteration.

$99 \times 49 \mathrm{~mm}(300 \times 300 \mathrm{DPI})$

This paper presented here as accepted for publication in Geophysics prior to copyediting and composition.

(C) 2021 Society of Exploration Geophysicists 


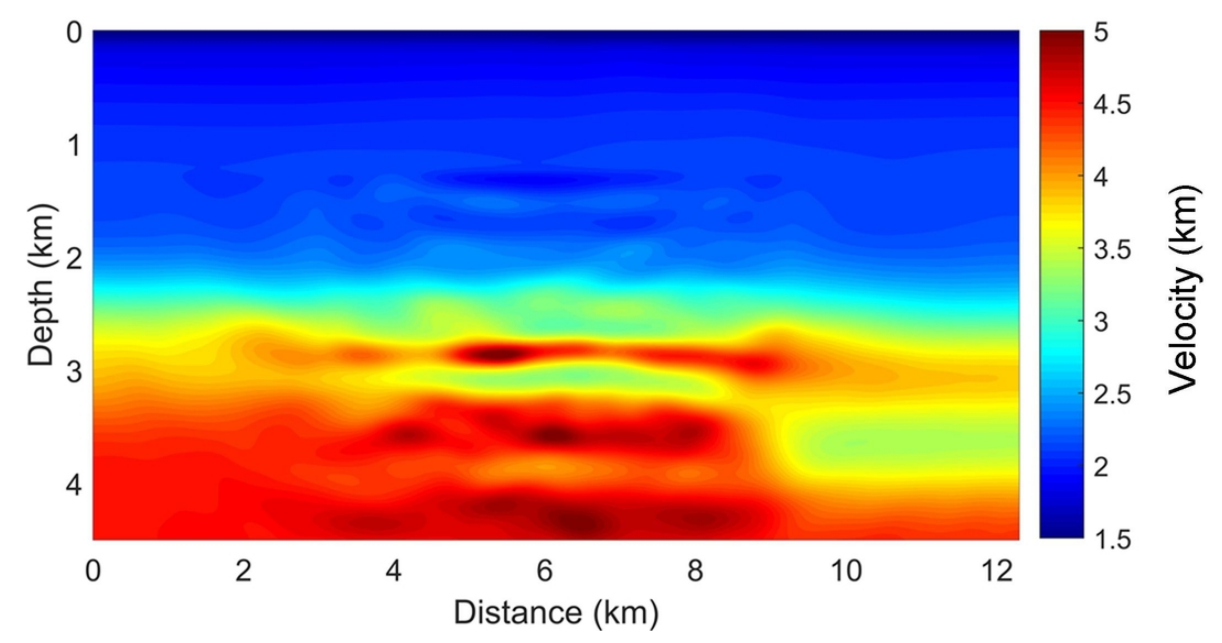

Figure 15: The inverted background velocity model using REWI.

$99 \times 49 \mathrm{~mm}(300 \times 300 \mathrm{DPI})$ 


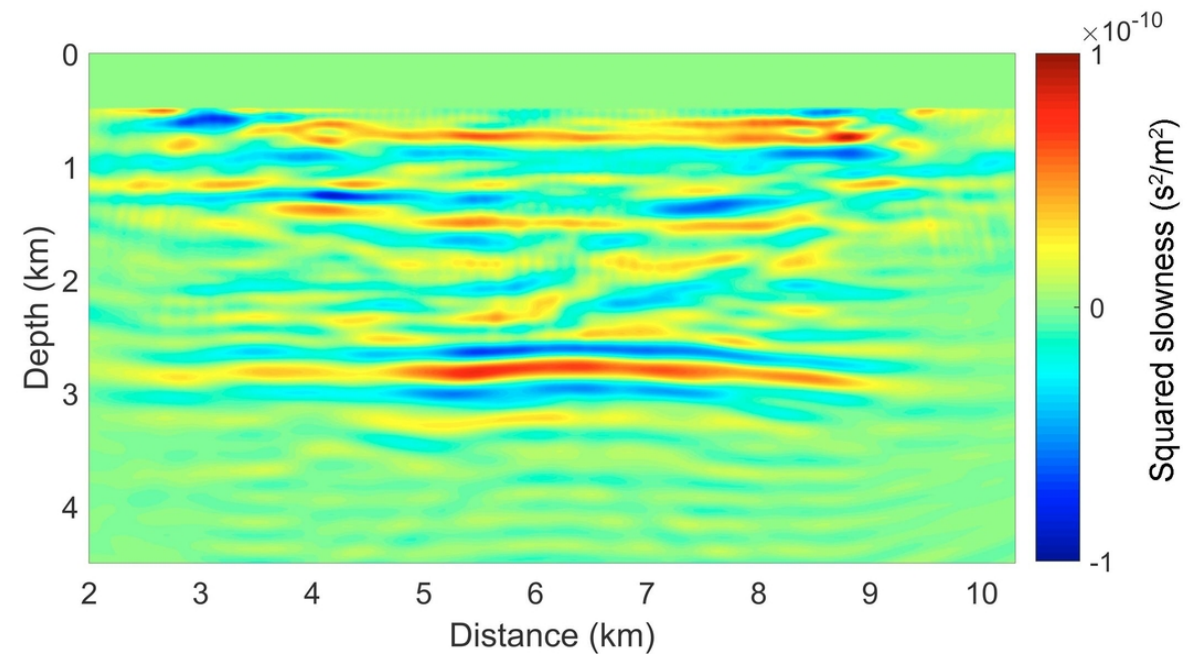

Figure 16: The inverted perturbation corresponding to the inverted background velocity at the last outer iteration.

$99 \times 49 \mathrm{~mm}(300 \times 300 \mathrm{DPI})$

This paper presented here as accepted for publication in Geophysics prior to copyediting and composition.

(C) 2021 Society of Exploration Geophysicists 
(a)

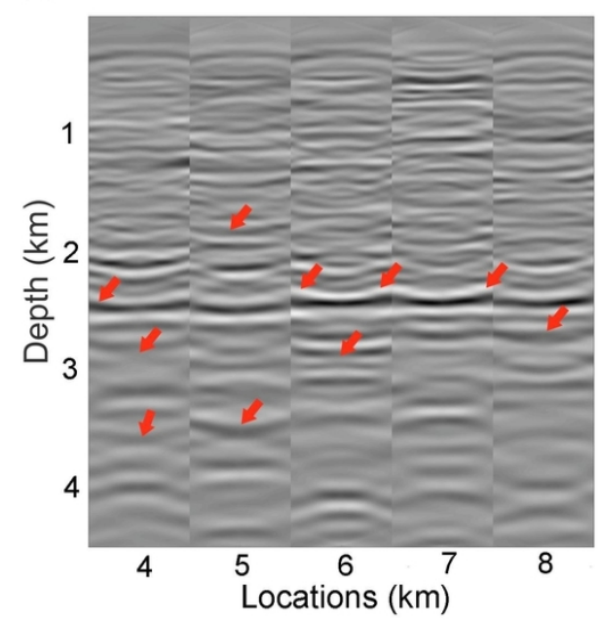

(b)

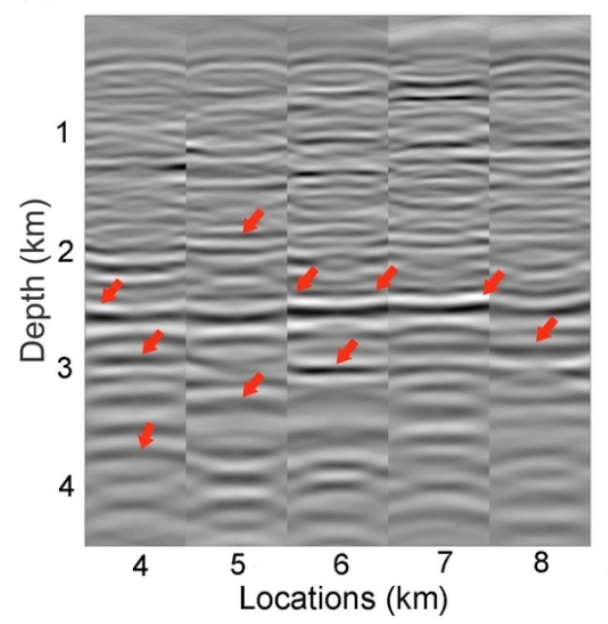

Figure 17: ADCIGs at $x=\{4,5,6,7,8\} \mathrm{km}$ corresponding to (a) the initial and (b) the REWI inverted velocity models. The arrows point to ADCIGs improvements in multiple areas.

$99 \times 49 \mathrm{~mm}(300 \times 300 \mathrm{DPI})$ 


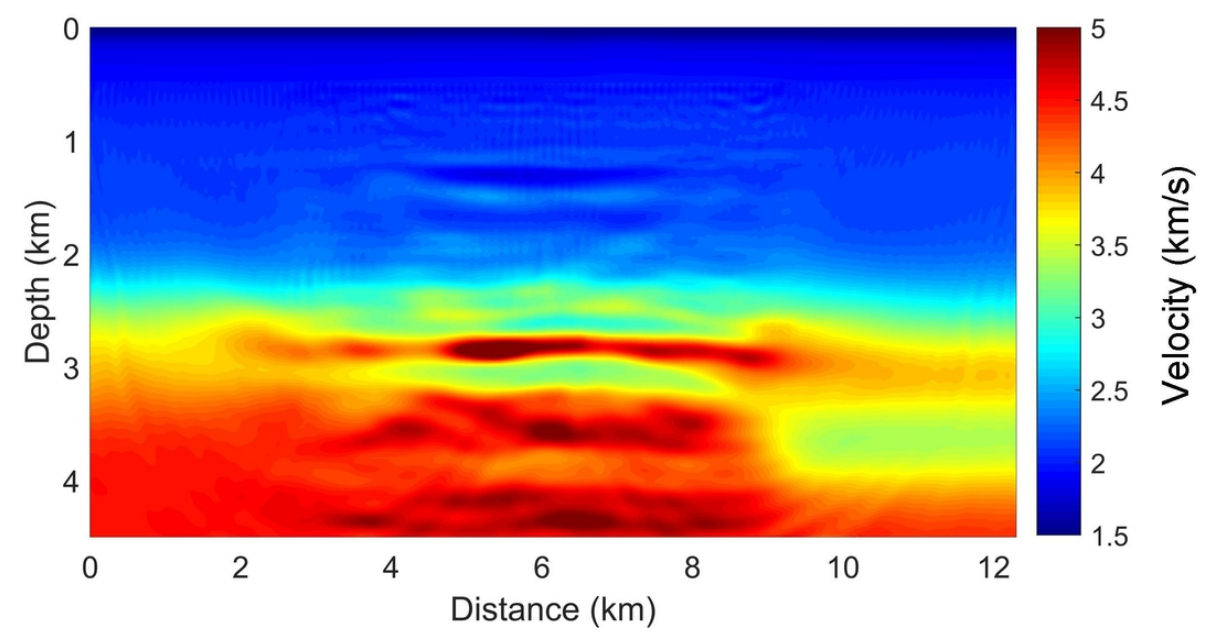

Figure 18: The sequential EWI inverted velocity model obtained using the inverted background model as the initial guess.

$99 \times 49 \mathrm{~mm}(300 \times 300$ DPI) 


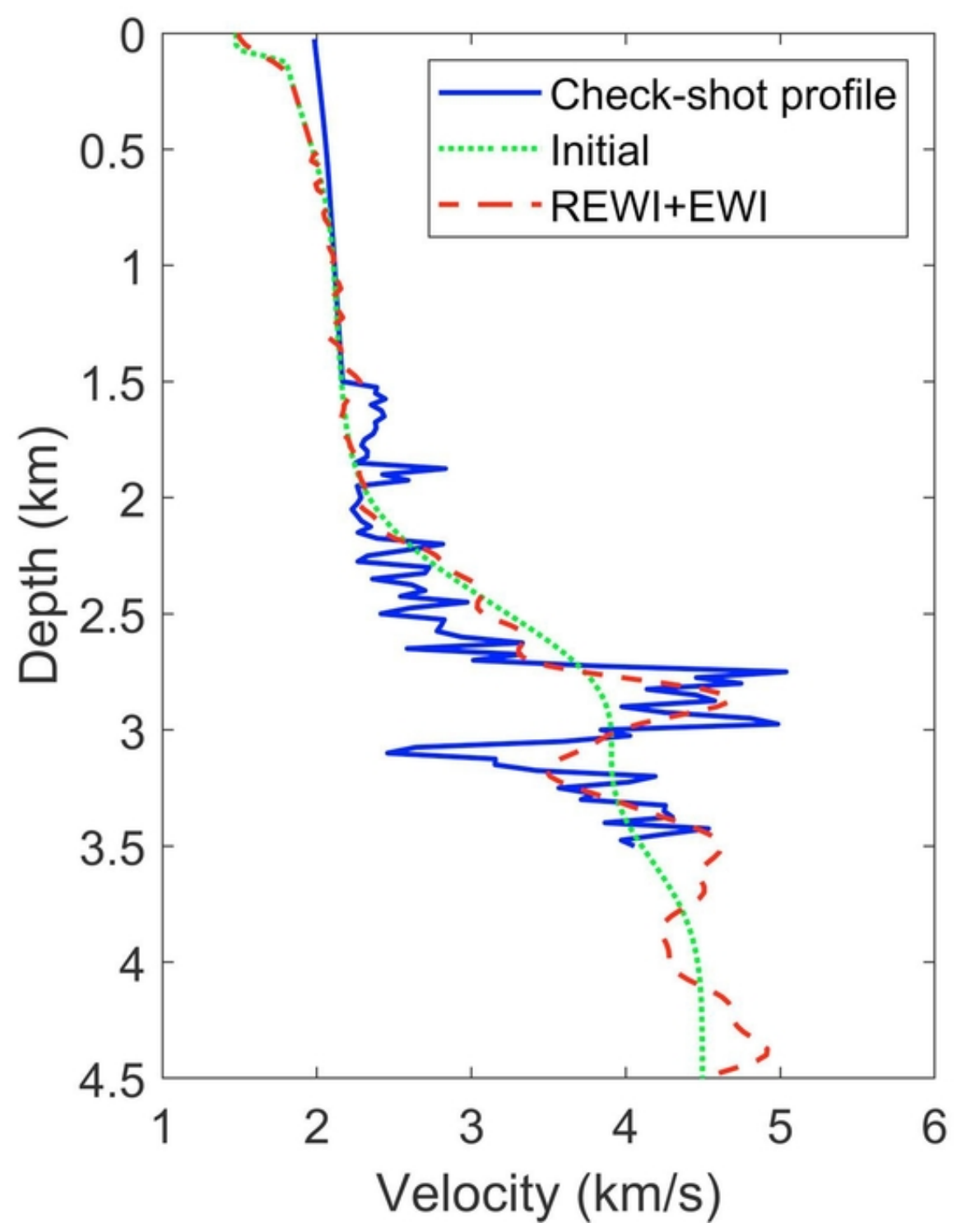

Figure 19: The vertical velocity profiles at location $x=8.24 \mathrm{~km}$ for the various inverted models compared to a check shot velocity profile at the same location.

$49 \times 62 \mathrm{~mm}(300 \times 300 \mathrm{DPI})$ 

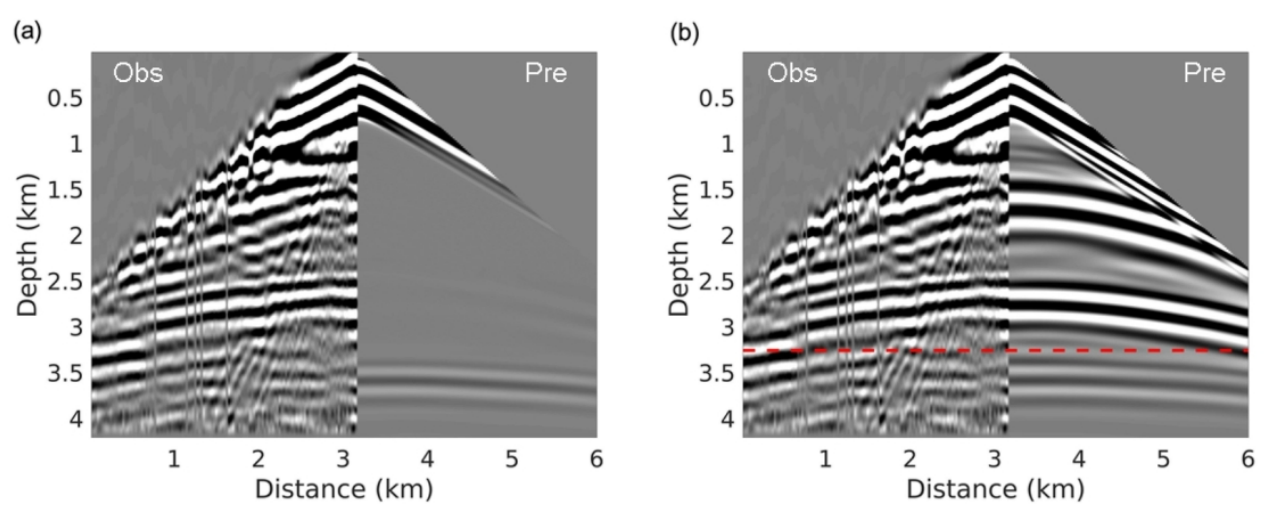

Figure 20: The shot gather at location $\mathrm{x}=6.175 \mathrm{~km}$ comparison between the filtered recoded data (left) and the predicted data (right) corresponding to the (a) initial and (b) inverted velocity models. A red dashed line in Figure $20 \mathrm{~b}$ demonstrates the data fitting the at far offset.

$$
149 \times 56 \mathrm{~mm}(300 \times 300 \mathrm{DPI})
$$



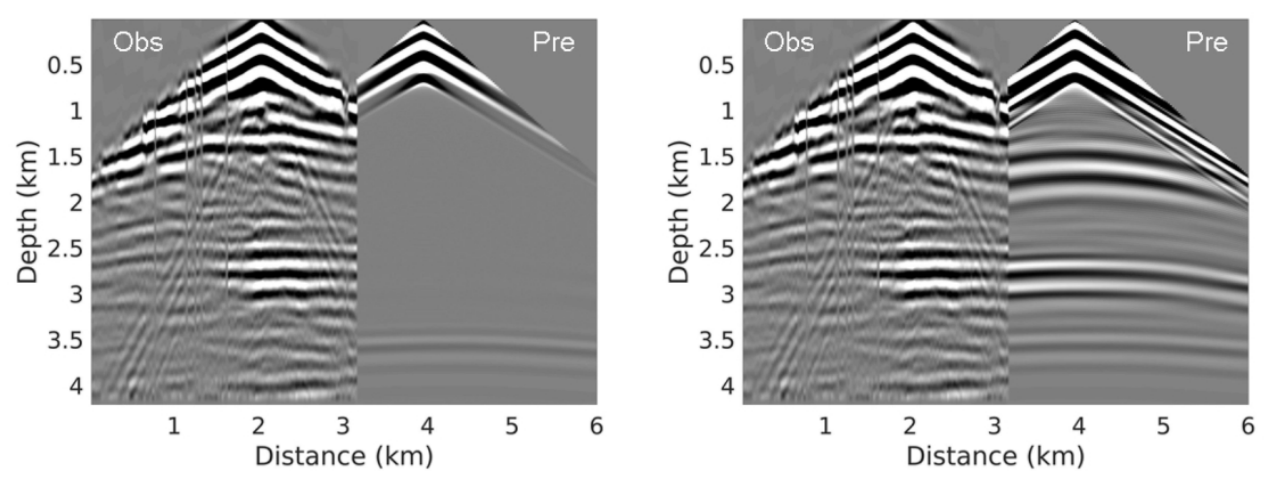

Figure 21: The shot gather at location $\mathrm{x}=5.175 \mathrm{~km}$ comparison between the filtered recoded data (left) and the predicted data (right) corresponding to the (a) initial and (b) inverted velocity models.

\section{$149 \times 56 \mathrm{~mm}(300 \times 300 \mathrm{DPI})$}


DATA AND MATERIALS AVAILABILITY

Data associated with this research are available and can be obtained by contacting the corresponding author. 